DOI 10.4171/JEMS/273

Shiri Artstein-Avidan · Vitali Milman

\title{
Hidden structures in the class of convex functions and a new duality transform
}

Received May 26, 2009

\begin{abstract}
Our main intention in this paper is to demonstrate how some seemingly purely geometric notions can be presented and understood in an analytic language of inequalities and then, with this understanding, can be defined for classes of functions and reveal new and hidden structures in these classes. One main example which we discovered is a new duality transform for convex non-negative functions on $\mathbb{R}^{n}$ attaining the value 0 at the origin (which we call "geometric convex functions") ${ }^{1}$ This transform, together with the classical Legendre transform, are essentially the only existing duality relations on this class of functions. Using these dualities we show that the geometric constructions of support and Minkowski functional may be extended, in a unique way, to the class of geometric log-concave functions, revealing hidden geometric structures on this class of functions.
\end{abstract}

\section{Introduction}

The Legendre transform is a classical and useful mathematical tool. It transforms (convex) functions on a vector space to functions on the dual space. The Legendre transform is related to projective duality and tangential coordinates in algebraic geometry, and to the construction of dual Banach spaces in analysis. It is often encountered in physics, for example in the definition of thermodynamic quantities, and plays a central role in several mathematical fields, including for example linear programming and game theory.

To formally define the Legendre transform $\mathcal{L}$ for a function $\phi: \mathbb{R}^{n} \rightarrow \mathbb{R} \cup\{\infty\}$, one first fixes a scalar product $\langle\cdot, \cdot\rangle$ on $\mathbb{R}^{n}$ (that is, a pairing between the space and the dual space). The Legendre transform $\mathcal{L}$ is then defined by

$$
(\mathcal{L} \phi)(x)=\sup _{y}(\langle x, y\rangle-\phi(y)) .
$$

It is an involution on the class of all lower-semicontinuous convex functions on $\mathbb{R}^{n}$, which we denote by $\operatorname{Cvx}\left(\mathbb{R}^{n}\right)$.

The above seems a very concrete definition, with a priori nothing special about it. However, it was demonstrated in [4] that this is the only order reversing involution on $\operatorname{Cvx}\left(\mathbb{R}^{n}\right)$. More precisely, we proved the following theorem:

Theorem 1. Let $\mathcal{T}: \operatorname{Cvx}\left(\mathbb{R}^{n}\right) \rightarrow \operatorname{Cvx}\left(\mathbb{R}^{n}\right)$ be a transform defined on the whole $\operatorname{Cvx}\left(\mathbb{R}^{n}\right)$ and such that for all $\phi, \psi \in \operatorname{Cvx}\left(\mathbb{R}^{n}\right)$,

S. Artstein-Avidan, V. Milman: School of Mathematical Science, Tel Aviv University, Ramat Aviv, Tel Aviv, 69978, Israel; e-mail: shiri@ post.tau.ac.il, milman@ post.tau.ac.il

1 See "Added in proof". 
1. $\mathcal{T} \mathcal{T} \phi=\phi$,

2. $\phi \leq \psi$ implies $T \phi \geq T \psi$.

Then there exists a constant $C_{0} \in \mathbb{R}$, a vector $v_{0} \in \mathbb{R}^{n}$ and a symmetric transformation $B \in \mathrm{GL}_{n}$ such that

$$
(\mathcal{T} \phi)(x)=(\mathcal{L} \phi)\left(B x+v_{0}\right)+\left\langle x, v_{0}\right\rangle+C_{0} .
$$

We called these two properties "abstract duality", so we may say that on the class $\operatorname{Cvx}\left(\mathbb{R}^{n}\right)$ there is, up to linear terms, only one duality transform, $\mathcal{L}$.

Note that the linear terms which appear are obvious additions coming from the choice of the origin and of a scalar product. In the rest of this note, we call two transforms "essentially" the same if they differ by similar linear terms. In these terms, a duality transform of $\operatorname{Cvx}\left(\mathbb{R}^{n}\right)$ (i.e., a bijection with properties 1 and 2 ) is essentially the Legendre transform.

Thus we see that the very concrete definition above is not ad hoc, and if one wants an order reversing transform on $\operatorname{Cvx}\left(\mathbb{R}^{n}\right)$ (the condition of involution can be relaxed somewhat) there is no other choice but to define the transform in this way.

There are two different approaches to duality in convex geometry. One is through the notion of the "polar body": Let

$$
\mathcal{K}_{0}\left(\mathbb{R}^{n}\right)=\left\{\text { all closed convex sets in } \mathbb{R}^{n} \text { containing } 0\right\}
$$

(we omit the notation $\mathbb{R}^{n}$ and simply write $\mathcal{K}_{0}$ when the dimension is clear). Fix a scalar product $\langle\cdot, \cdot\rangle$ in $\mathbb{R}^{n}$. Then the polar body of $K \in \mathcal{K}_{0}$ is defined by

$$
K^{\circ}=\left\{x \in \mathbb{R}^{n}:\langle x, y\rangle \leq 1 \text { for all } y \in K\right\} \in \mathcal{K}_{0} .
$$

In the other approach, one defines the supporting function of $K, h_{K}(x)=\sup _{y \in K}\langle x, y\rangle$, which is 1-homogeneous, non-negative, and convex. Clearly the approaches are equivalent as $K^{\circ}=\left\{x: h_{K}(x) \leq 1\right\}$. Define $\mathcal{H}_{0}$ to be the family of all 1-homogeneous, non-negative, and convex functions.

We embed $\mathcal{K}_{0}\left(\mathbb{R}^{n}\right)$ in $\operatorname{Cvx}\left(\mathbb{R}^{n}\right)$ by

$$
K \mapsto 1_{K}^{\infty}= \begin{cases}0, & x \in K, \\ +\infty, & \text { otherwise }\end{cases}
$$

The polarity map $P:\left\{1_{K}: K \in \mathcal{K}_{0}\right\} \rightarrow\left\{1_{K}: K \in \mathcal{K}_{0}\right\}$ given by $P\left(1_{K}^{\infty}\right)=1_{K^{\circ}}^{\infty}$ is essentially the only order reversing bijection on this class, and the support map $S:\left\{1_{K}\right.$ : $\left.K \in \mathcal{K}_{0}\right\} \rightarrow \mathcal{H}_{0}$ given by $S(K)=h_{K}$ is essentially the only order reversing bijection between these classes (see below for exact statements and references).

In this paper, we show that when one considers the class $\operatorname{Cvx}_{0}\left(\mathbb{R}^{n}\right)$ of non-negative lower-semicontinuous convex functions $f: \mathbb{R}^{n} \rightarrow[0, \infty]$ which take the value 0 at 0 , there is a unique extension of $S$ to this class, which is the Legendre transform, and, more surprisingly, there is a unique extension of the geometric polarity $P$ to $\operatorname{Cvx}_{0}\left(\mathbb{R}^{n}\right)$. This last extension is given by a new duality transform on $\operatorname{Cvx}_{0}\left(\mathbb{R}^{n}\right)$, which we call $\mathcal{A}$. So, the Legendre transform which we usually call "duality" for convex functions actually represents the support map on this class, whereas geometric polarity for convex functions is 
represented by the new map $\mathcal{A}$. Note that the support map/Legendre transform is defined on all of $\operatorname{Cvx}\left(\mathbb{R}^{n}\right)$, in the same way as the support map is actually defined for all convex bodies, not necessarily including 0 , whereas the same is not true for $\mathcal{A}$ (or, correspondingly, for geometric polarity).

Thus, when considering the subclass $\operatorname{Cvx}_{0}\left(\mathbb{R}^{n}\right)$ of $\operatorname{Cvx}\left(\mathbb{R}^{n}\right)$, instead of the whole class, for questions of duality, the situation is drastically different. We name $\mathrm{Cvx}_{0}\left(\mathbb{R}^{n}\right)$ the "geometric convex functions". It is an invariant subclass of $\operatorname{Cvx}\left(\mathbb{R}^{n}\right)$ under $\mathcal{L}$, so that $\mathcal{L}$ acts as abstract duality on it (that is, an order reversing involution). However, one now has another, very different, transform $\mathcal{A}$ which acts on $\mathrm{Cvx}_{0}\left(\mathbb{R}^{n}\right)$ as abstract duality (and which cannot be extended to all of $\left.\operatorname{Cvx}\left(\mathbb{R}^{n}\right)\right)$. This new transform has the potential of being just as useful as $\mathcal{L}$ (and, as we will see, in some cases more useful) whenever the class one is interested in does not consist of all convex functions, but only non-negative ones with $f(0)=0$. We define $\mathcal{A}$ in Section 2 , and name it "geometric duality", as we show that it is more suitable for some geometric problems.

We also show that there still remains a uniqueness property, namely these two transforms, $\mathcal{A}$ and $\mathcal{L}$, are essentially (up to linear terms) the only duality transforms on $\operatorname{Cvx}_{0}\left(\mathbb{R}^{n}\right)$. This is stated in Theorem 2 proven in Section 6 .

The new transform $\mathcal{A}$ has interesting geometric properties, some resembling the properties of the well studied Legendre transform $\mathcal{L}$, and some quite different. We also study the order preserving transform $\mathcal{L A}$, which is essentially the only injective order preserving (in both directions) transform on $\mathrm{Cvx}_{0}\left(\mathbb{R}^{n}\right)$ other than the identity transform Id. In particular, we will see that this implies the commutation relation $\mathcal{L} \mathcal{A}=\mathcal{A L}$. Since this transform also plays a special role in this paper, we give it a name, $\mathcal{J}$, and study its properties. We will see below that an appropriate name for this transform is "gauge transform", and it gives an interpretation of what it could mean to be the gauge function of a convex, or, after a suitable correction, log-concave, function (rather than a convex body).

The new transform $\mathcal{A}$, together with the Legendre transform $\mathcal{L}$, allow us to see new "hidden" structures on the class of convex functions on $\mathbb{R}^{n}$. The classical notion of "support function" and "Minkowski functional" for a convex set play a central role in convexity theory and correspondingly in geometric inequalities. The standard definition of these two notions uses very strongly the geometry of the convex body to which they are related (the support function is defined using supporting hyperplanes of the body, and the Minkowski functional is defined to be the 1-homogeneous function with "unit ball" equaling the body). After showing that the two notions may be alternatively defined in the language of order preserving or order reversing transformations, a language equally applicable to functions as to bodies, these two central notions may be extended to the class of geometric log-concave functions in a very natural (and, as we will see, unique) way. We believe, and in some cases know already, that this extension will lead to functional versions of many geometric inequalities, which is one of the goals of such extensions. However this is outside the realm of this paper, and will be developed elsewhere. (We remark that some such extensions for operations involving $\mathcal{L}$ were done earlier: see [2], [13] and [12]; in particular, the Santaló inequality was proved in [2] for the duality involving $\mathcal{L}$. A variant of the Santaló inequality which involves $\mathcal{A}$ also exists, and will be presented in a forthcoming paper.) 
The paper is organized as follows. In Section 2 we define the new transform $\mathcal{A}$, discuss some of its simple properties, and also very briefly discuss the transform $\mathcal{J}=\mathcal{L} \mathcal{A}$. In Section 3 we state the main uniqueness theorems, for $\mathcal{L}$ and $\mathcal{A}$, and for $\mathcal{J}$, and a simple corollary. In Section 4 we give the geometric interpretation of the transform $\mathcal{A}$. Section 5 includes some more calculus of the three transforms, and an elaboration of simple properties of $\mathcal{J}$. In Section 6 we provide the full proof of the uniqueness result, which is divided into many steps. In Section 7 we restate some of the theorems in the language of log-concave functions, where there is a new geometric meaning to them, and some new geometric operations can be defined and analyzed. In Section 8 we give a simple example of a class which exhibits no duality transform. In Section 9 we discuss a class for which only $\mathcal{A}$ acts as duality, and in the last section 10 we discuss the geometric operations of support map and Minkowski map. We show that they can be defined by inequalities rather than concrete geometric constructions, and therefore can be naturally extended, and in a unique way, to the class $\operatorname{Cvx}_{0}\left(\mathbb{R}^{n}\right)$.

\section{The new transforms: definition and first properties}

\subsection{Defining $\mathcal{A}$}

Consider the following transform, defined on $\operatorname{Cvx}_{0}\left(\mathbb{R}^{n}\right)$ :

$$
(\mathcal{A} f)(x)= \begin{cases}\sup _{\left\{y \in \mathbb{R}^{n}: f(y)>0\right\}} \frac{\langle x, y\rangle-1}{f(y)} & \text { if } x \in\left\{f^{-1}(0)\right\}^{\circ}, \\ +\infty & \text { if } x \notin\left\{f^{-1}(0)\right\}^{\circ}\end{cases}
$$

(with the convention $\sup \emptyset=0$ ). Note that although $\mathcal{A}$ implicitly depends on the dimension $n$, we do not complicate notation, and use the same letter $\mathcal{A}$ for all dimensions.

Note also that for $x \notin\{f(y)=0\}^{\circ}$ (the second case), there is some $y \in \mathbb{R}^{n}$ with $f(y)=0$ and with $\langle x, y\rangle>1$, so that formally, we can simply let

$$
(\mathcal{A} f)(x)=\sup _{y \in \mathbb{R}^{n}} \frac{\langle x, y\rangle-1}{f(y)}
$$

where we define $\frac{t}{0}=+\infty$ (and $\frac{0}{0}=0$, say). The only exception is that for this definition to work for the identically 0 function we would need to set $\overline{\overline{0}}=(\overline{\overline{0}})_{+}=0$.

\subsection{Simple properties of $\mathcal{A}$}

1. Order reversing. The transform $\mathcal{A}$ is order reversing: if $f \leq g$ then $\mathcal{A} f \geq \mathcal{A g}$. Indeed, if $f \leq g$ then $\{y: g(y)=0\} \subset\{y: f(y)=0\}$ and so $\{y: f(y)=0\}^{\circ} \subset\{y$ : $g(y)=0\}^{\circ}$. It is thus clear from the definition that $\mathcal{A} f \geq \mathcal{A} g$.

2. Involution. One may directly compute that for all $f \in \operatorname{Cvx}_{0}\left(\mathbb{R}^{n}\right)$ we have $\mathcal{A} \mathcal{A} f=f$. However, this will follow very easily from the geometric interpretation of $\mathcal{A}$ in Section 4 below. 
These first two properties, when valid for a mapping from a class $\mathcal{S}$ of functions to itself, are what we call "abstract duality" on the class $\mathcal{S}$ (see for example the papers [3] or [4]). The two properties are valid, as noted above, also for the Legendre transform $\mathcal{L}$. Thus we see that when one restricts the class $\operatorname{Cvx}\left(\mathbb{R}^{n}\right)$ by requiring the functions to be nonnegative and attain the value 0 at 0 , then unlike the case of $\operatorname{Cvx}\left(\mathbb{R}^{n}\right)$, on $\operatorname{Cvx}_{0}\left(\mathbb{R}^{n}\right)$ there are at least two, very different (as will be clear from the properties below) transforms.

Let us observe some more simple properties of $\mathcal{A}$.

3. Homogeneity. For $a>0$,

$$
(\mathcal{A}(a f))(y)=(1 / a)(\mathcal{A} f)(y)
$$

and letting $f_{a}(x)=f(x / a)$ we have

$$
\left(\mathcal{A} f_{a}\right)(y)=(\mathcal{A} f)_{1 / a}(y) .
$$

Note that for $\mathcal{L}$ we have $\mathcal{L}(a f)=a(\mathcal{L} f)_{a}$ and $\mathcal{L}\left(f_{a}\right)=(\mathcal{L} f)_{1 / a}$.

4. Action on norms. For any norm $\|\cdot\|$, we have

$$
(\mathcal{A}\|\cdot\|)(x)=\|x\|^{*},
$$

where $\|x\|^{*}=\sup \{\langle x, y\rangle:\|y\| \leq 1\}$ is the dual norm. Indeed,

$$
(\mathcal{A}\|\cdot\|)(x)=\sup _{y \neq 0} \frac{\langle x, y\rangle-1}{\|y\|}=\sup _{y \in S^{n-1}} \sup _{t>0} \frac{t\langle x, y\rangle-1}{t\|y\|}=\sup _{y \in S^{n-1}} \frac{\langle x, y\rangle}{\|y\|}=\|x\|^{*} .
$$

The same is true also when $\|\cdot\|$ is a generalized norm, that is, it need not be symmetric and may assume the values 0 and $+\infty$.

From here onwards we let $\|\cdot\|$ stand for such a generalized norm, that is, a positivelyhomogeneous convex function with values in $\mathbb{R}_{\geq 0} \cup\{+\infty\}$ (so that its unit ball is some closed convex set $K$, possibly unbounded, containing 0 , possibly at its boundary, and $\|\cdot\|=\|\cdot\|_{K}$ is called the Minkowski functional of the body $K$ ).

Note that for $\mathcal{L}$ we have

$$
(\mathcal{L}\|\cdot\|)(x)=1_{K^{\circ}}^{\infty}(x)
$$

where $1_{T}^{\infty}$, as defined also in the introduction, stands for the convex function which is 0 on the convex body $T$ and $+\infty$ elsewhere, and $K^{\circ}$ is the polar body of the unit ball of $\|\cdot\|$, namely

$$
K^{\circ}=\{x:\langle x, y\rangle \leq 1 \forall\|y\| \leq 1\}=\left\{x:\|x\|^{*} \leq 1\right\} .
$$

5. Action on powers of norms. For every power $p>1$ we have

$$
\left(\mathcal{A}\left(\|\cdot\|^{p}\right)\right)(x)=\frac{(p-1)^{p-1}}{p^{p}}\left(\|x\|^{*}\right)^{p},
$$

where the computation is almost identical to the case of $p=1$. In particular,

$$
\mathcal{A}\left(\|\cdot\|^{2} / 2\right)(x)=\left(\|x\|^{*}\right)^{2} / 2
$$

for any generalized norm. 
Note that for $\mathcal{L}$ we have $\mathcal{L}\left(p^{-1}\|\cdot\|^{p}\right)=q^{-1}\left(\|\cdot\|^{*}\right)^{q}$ where $1 / p+1 / q=1$, that is, $\mathcal{A}$ coincides with $\mathcal{L}$ on the subclass of 2-homogeneous functions, but for $p$-homogeneous functions with $p \neq 2$ there is a dramatic difference between the two duality transforms.

We remark that when the class consists of positive-2-homogeneous functions (squares of generalized norms), which is an invariant subset with respect to both transforms ( $\mathcal{A}$ and $\mathcal{L}$ coincide on this subset), there is again just one duality transform, the standard duality of norms (for exact definitions and a proof of this result see [5]).

6. Action on indicators of convex sets. With the same notation as before, we have

$$
\mathcal{A} 1_{K}^{\infty}=1_{K^{\circ}}^{\infty}
$$

Again, this is very different from the behavior of $\mathcal{L}$ (since $\mathcal{L} 1_{K}^{\infty}=\|\cdot\|^{*}$ with $\|\cdot\|^{*}$ denoting the norm with unit ball $K^{\circ}$ ), and so we see that $(3)$ is a property that cannot hold for any duality transform on the whole of $\operatorname{Cvx}\left(\mathbb{R}^{n}\right)$, but it does hold for $\mathcal{A}$ which is a duality transform on the restricted class $\operatorname{Cvx}_{0}\left(\mathbb{R}^{n}\right)$. Actually, 3 shows that $\mathcal{A}$ indeed extends the standard geometric duality for convex sets to the functional level, and gives a first justification for the choice of the name "geometric duality" for $\mathcal{A}$.

7. Action on norm-radial functions. For a convex function $f(t)$ on $\mathbb{R}^{+}$with $f(0)=0$ and for any norm $\|\cdot\|$ we have $\mathcal{A}(f(\|\cdot\|))=(\mathcal{A} f)\left(\|\cdot\|^{*}\right)$ where the first transform is on $\mathbb{R}^{n}$ and the second is the one-dimensional one, on the half-line $\mathbb{R}^{+}$(one may define $\mathcal{A}$ on a cone instead of on the full linear space; we do this for $\mathbb{R}^{+}$in Section 6.6. Note that the same is true for $\mathcal{L}$, namely $\mathcal{L}(f(\|\cdot\|))=(\mathcal{L} f)\left(\|\cdot\|^{*}\right)$.

8. Kernel and support. For a convex lower-semicontinuous function $f: \mathbb{R}^{n} \rightarrow \mathbb{R}^{+} \cup$ $\{+\infty\}$ we denote by $\operatorname{Ker}(f)$ the subset on which $f$ equals 0 (this is always a closed convex set), and by $\operatorname{supp}(f)$ the subset on which $f$ is finite (this is a convex set, but may not be closed; note that we do not take the closure of this set, though it is sometimes more natural). That is,

$$
\operatorname{Ker}(f)=\{x: f(x)=0\}, \quad \operatorname{supp}(f)=\{x: f(x)<\infty\} .
$$

Then

$$
(\operatorname{Ker}(\mathcal{A} f))^{\circ}=\overline{\operatorname{supp}(f)} \text { and }(\operatorname{supp}(\mathcal{A} f))^{\circ}=\operatorname{Ker}(f) .
$$

Clearly, by the involution property of $\mathcal{A}$, we see that $\mathcal{A}$ is a bijection between all functions with $\operatorname{Ker}(f)=K$ and all functions such that $\overline{\operatorname{supp}(f)}=K^{\circ}$.

For the Legendre transform we have the following property, slightly more difficult to formulate:

$$
(\mathcal{L} f)(x) \geq \sup _{\{y: f(y)=0\}}\langle x, y\rangle=\|x\|_{(\operatorname{Ker} f)^{\circ}},
$$

and if $\mathcal{L} f \geq\|x\|_{T}$ for every $x$, then $T^{\circ} \subset \operatorname{Ker} f$. (That is, $\|x\|_{(\operatorname{Ker} f)^{\circ}}$ is the largest norm below $\mathcal{L} f$.) Similarly, the set $(\operatorname{supp} f)^{\circ}$ is the largest set satisfying $\mathcal{L} f \leq$

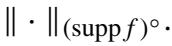




\subsection{Order preserving maps and the gauge transform}

Instead of discussing $\mathcal{A}$ and $\mathcal{L}$, we may discuss order preserving transforms on $\operatorname{Cvx}_{0}\left(\mathbb{R}^{n}\right)$. One example of such a transform is, of course, the identity transform, or, more generally, the transform which maps a function to a constant multiple of its composition with any non-singular linear transformation. This kind of transform is bijective and order preserving, as is its inverse, and we call such a transform "essentially identity".

However, when one has (as we do) two order reversing transformations, the two can be composed to yield an order preserving transformation, and if the two original transforms differ by more than mere linear terms, the new transform will be "essentially" different from the identity.

In fact, if we eliminate the condition of involution, and only require the transforms to be bijective, the discussion of order preserving transformations is equivalent to the discussion of order reversing ones, since any order reversing transform, when composed, say, with $\mathcal{L}$, turns into an order preserving one, and vice versa. In particular, a complete description of order reversing maps on $\mathrm{Cvx}_{0}\left(\mathbb{R}^{n}\right)$ would also provide a complete description of order preserving maps, as we will indicate below. (In fact, in most of our proofs it will be easier to work with order preserving maps).

We thus define a new order preserving transformation by

$$
\mathcal{J} f=\mathcal{L} \mathcal{A} f .
$$

Of course, we may also define an order preserving transformation by $f \mapsto \mathcal{A L} f$, and it can be computed and seen to give the exact same result, a fact which will follow easily, with hardly any need for computations, from the uniqueness theorem below. This is explained in Section 3

The formula for $\mathcal{J}$ can be computed, and has the following form:

$$
(\mathcal{J} f)(x)=\inf \{r>0: f(x / r) \leq 1 / r\}
$$

(where the infimum of an empty set is $+\infty$ ).

It might not be a priori clear why the resulting function is convex. This will, however, be immediate from the geometric interpretation for $\mathcal{J}$ which we will give below (and can also be verified directly). We delay the discussion of the simple properties of $\mathcal{J}$ to Section 5, but the reader may easily find its properties corresponding to those of $\mathcal{A}$ above. One of these properties, which we mention here and elaborate on later, is that this transform, although quite different from the identity, still shares with it the very special property, that it acts "ray-wise", i.e., its values on a given ray depend only on the values of $f$ on this ray.

In other words, the way $\mathcal{L}$ "mixes" $\mathbb{R}^{n}$ and the way $\mathcal{A}$ does, cancel each other, so that $\mathcal{J}=\mathcal{L} \mathcal{A}$ acts on each ray separately: If for some ray $R=\mathbb{R}^{+} y$ we have $\left.f\right|_{R}=\left.g\right|_{R}$ then also $\left.\mathcal{J} f\right|_{R}=\left.\mathcal{J} g\right|_{R}$.

Note that

$$
\mathcal{J} 1_{K}^{\infty}=\mathcal{L} \mathcal{A} 1_{K}^{\infty}=\mathcal{L} 1_{K^{\circ}}^{\infty}=\|\cdot\|_{K},
$$

which explains why the name "gauge transform" or "gauge map" is appropriate for $\mathcal{J}$. 


\section{The uniqueness theorem}

It turns out that the second form, $\mathcal{A}$, of duality for the class $\operatorname{Cvx}_{0}\left(\mathbb{R}^{n}\right)$ is the only option other than $\mathcal{L}$ :

Theorem 2. Let $n \geq 2$. Any order reversing involution $\mathcal{T}: \operatorname{Cvx}_{0}\left(\mathbb{R}^{n}\right) \rightarrow \operatorname{Cvx}_{0}\left(\mathbb{R}^{n}\right)$ is of the form either $\mathcal{T} f=(\mathcal{L} f) \circ B$ or $\mathcal{T} f=C_{0}(\mathcal{A} f) \circ B$ for some symmetric $B \in \mathrm{GL}_{n}$ and $C_{0}>0$.

In the one-dimensional version a similar theorem holds; however, because $\operatorname{Cvx}_{0}(\mathbb{R})$ is a direct product of $\operatorname{Cvx}_{0}\left(\mathbb{R}^{+}\right)$with itself (the point 0 , when $f(0)=0$, disconnecting the two parts), the mapping can act in two different ways on each part, and can also switch the two parts. So, there are eight possible variants, listed in the following one-dimensional theorem which in turn will be a consequence of the theorem for $\operatorname{Cvx}_{0}\left(\mathbb{R}^{+}\right)$which is stated as Theorem 17 in Section 6.6 below.

Theorem 3. Any order reversing involution $\mathcal{T}: \operatorname{Cvx}_{0}(\mathbb{R}) \rightarrow \operatorname{Cvx}_{0}(\mathbb{R})$ has one of the following forms, for some $\alpha, \beta, \gamma>0$ :

1. $(\mathcal{T} f)(x)=\alpha(\mathcal{A} f)(\beta x)$,

2. $(\mathcal{T} f)(x)=(\mathcal{L} f)(\beta x)$

3. $(\mathcal{T} f)(x)=\alpha(\mathcal{A} f)(-\beta x)$,

4. $(\mathcal{T} f)(x)=(\mathcal{L} f)(-\beta x)$,

5. $(\mathcal{T} f)(x)=\alpha(\mathcal{A} f)(\beta x)$ for $x>0$ and $(\mathcal{L} f)(\gamma x)$ for $x<0$,

6. $(\mathcal{T} f)(x)=(\mathcal{L} f)(\beta x)$ for $x>0$ and $\alpha(\mathcal{A} f)(\gamma x)$ for $x<0$,

7. $(\mathcal{T} f)(x)=\alpha(\mathcal{A} f)(-\beta x)$ for $x>0$ and $(\mathcal{L} f)(-\gamma x)$ for $x<0$,

8. $(\mathcal{T} f)(x)=(\mathcal{L} f)(-\beta x)$ for $x>0$ and $\alpha(\mathcal{A} f)(-\gamma x)$ for $x<0$.

As in the case of $\operatorname{Cvx}\left(\mathbb{R}^{n}\right)$, the condition of involution may be replaced by the weaker condition that both $\mathcal{T}$, which we assume to be 1-1 and onto, and its inverse, are order reversing, in which case we get the same conclusion but where $B$ need not be symmetric.

Remark. When we write below that a bijective transformation $\mathcal{T}$ is order preserving (respectively reversing) "in both directions", we mean that both $\mathcal{T}$ and $\mathcal{T}^{-1}$ are order preserving (respectively reversing).

Theorem 4. Let $n \geq 2$. Any bijective transform $\mathcal{T}: \operatorname{Cvx}_{0}\left(\mathbb{R}^{n}\right) \rightarrow \operatorname{Cvx}_{0}\left(\mathbb{R}^{n}\right)$ which is order reversing in both directions is either of the form $\mathcal{T} f=C_{0}(\mathcal{L} f) \circ B$ or of the form $\mathcal{T} f=C_{0}(\mathcal{A} f) \circ B$ for some $B \in \mathrm{GL}_{n}$ and $C_{0}>0$.

For order preserving maps we of course have a similar fact, and Theorem 4 will be derived as a corollary from the following

Theorem 5. Let $n \geq 2$. Any bijective transform $\mathcal{T}: \operatorname{Cvx}_{0}\left(\mathbb{R}^{n}\right) \rightarrow \operatorname{Cvx}_{0}\left(\mathbb{R}^{n}\right)$ which is order preserving in both directions is either of the form $\mathcal{T} f=C_{0} f \circ B$ or of the form $\mathcal{T} f=C_{0}(\mathcal{J} f) \circ B$ for some $B \in \mathrm{GL}_{n}$ and $C_{0}>0$. 
We leave it to the interested reader to state the one-dimensional results when the condition of involution is relaxed, and when order reversal is exchanged with order preservation.

We end this section with a simple corollary. One can compute directly that $\mathcal{L} \mathcal{A}=\mathcal{A L}$. This is completely equivalent to the fact that $\mathcal{J}$ is an involution, since $\mathcal{L} \mathcal{A} \mathcal{A} \mathcal{L}=\mathcal{L} \mathcal{L}$ $=\mathrm{Id}$. However, one can also see this as a corollary from Theorem 5 . Since $\mathcal{A L}$ is order preserving, it must be either of the form $C_{0} \mathcal{J} f \circ B$ or $C_{0} f \circ B$. However, since we know that $\mathcal{A L}\|\cdot\|_{K}=1_{K}^{\infty}$ for every $K$, it must be of the first form, and also then clearly $B=\operatorname{Id}_{n}$, and since $\mathcal{A} \mathcal{L} 1_{K}^{\infty}=\|\cdot\|_{K}$, it follows that $C_{0}=1$. We have thus shown

Corollary 6. For any function $f \in \mathrm{Cvx}_{0}\left(\mathbb{R}^{n}\right)$ we have

$$
\mathcal{J} f=\mathcal{L} \mathcal{A} f=\mathcal{A L} f
$$

or, in other words, $\mathcal{J} \mathcal{J}=\mathrm{Id}$.

\section{Geometric interpretations}

Some of the basic properties of $\mathcal{A}$ (and also of $\mathcal{J}$ ) can be understood more clearly when one sees the geometry behind them. In fact, the geometric interpretation indicates that $\mathcal{A}$ is perhaps a more natural candidate for "duality" than $\mathcal{L}$ (this can also be seen from property 6 of $\mathcal{A}$ in Section 2.2.

We begin with a short introduction. There are two different and natural ways to associate to a function $\phi \in \operatorname{Cvx}_{0}\left(\mathbb{R}^{n}\right)$ a closed convex set in $\mathbb{R}^{n+1}$ which is contained in the upper half-space $\mathbb{R}^{n} \times \mathbb{R}_{\geq 0}$, and contains the ray $\{0\} \times \mathbb{R}_{\geq 0}$. One way is to consider the epigraph of the function,

$$
\operatorname{epi}(\phi)=\{(x, r): r \geq \phi(x)\} .
$$

Another is to extend $\phi$ from the hyperplane $\left\{(x, 1): x \in \mathbb{R}^{n}\right\}$ homogeneously for $(x, r)$ with $r>0$, and to take the closure of the unit ball of the generalized norm obtained by this procedure. This gives

$$
K_{\phi}=\overline{\left\{(x, y) \in \mathbb{R}^{n} \times \mathbb{R}: y>0 \text { and } \phi(x / y) \leq 1 / y\right\}} .
$$

The resemblance of the definition of this set to the definition of $\mathcal{J}$ is no accident.

Note that duality-and-reflection acts invariantly on this class of convex subsets of $\mathbb{R}^{n+1}$, namely if $K$ is in the class then so is

$$
\left\{(x,-r):(x, r) \in K^{\circ}\right\}=: \operatorname{ref}\left(K^{\circ}\right) .
$$

Here for $S \subset \mathbb{R}^{n} \times \mathbb{R}$, we let $\operatorname{ref}(S)$ stand for its reflection with respect to the subspace spanned by the first $n$ coordinates.

It is an easy exercise to verify that the reflection of the polar of epi $(\phi)$ with respect to $\mathbb{R}^{n}$ is the epigraph of $\mathcal{A} \phi$, that is,

$$
\operatorname{epi}(\mathcal{A} \phi)=\left\{(x,-r) \in \mathbb{R}^{n} \times \mathbb{R}_{\geq 0}:(x, r) \in(\operatorname{epi}(\phi))^{\circ}\right\}=\operatorname{ref}\left((\operatorname{epi}(\phi))^{\circ}\right) .
$$


It is also not difficult to check that the same is true for the second description, that is,

$$
K_{\mathcal{A} \phi}=\left\{(x,-r) \in \mathbb{R}^{n} \times \mathbb{R}_{\geq 0}:(x, r) \in K_{\phi}^{\circ}\right\}=\operatorname{ref}\left(K_{\phi}^{\circ}\right) .
$$

In particular, the involution property of $\mathcal{A}$ follows immediately from either of the two descriptions.

So, if we stick to one such mapping between $\operatorname{Cvx}_{0}\left(\mathbb{R}^{n}\right)$ and this class of convex sets, then up to reflection, $\mathcal{A}$ is the classical convex-geometric duality for sets.

The connection between the two descriptions is given by $\mathcal{J}$, namely the relation epi $(\varphi)=K_{\psi}$ gives an order preserving correspondence $\psi \leftrightarrow \varphi$ (which is an involution), and this is precisely $\varphi=\mathcal{J} \psi$. That is,

$$
\mathcal{J} \phi=\mathcal{L} \mathcal{A} \phi=\operatorname{epi}^{-1}\left(K_{\phi}\right) \quad \text { and } \quad K_{\mathcal{L} \mathcal{A} \phi}=\operatorname{epi}(\phi) .
$$

We remark that from this one gets a geometric interpretation for the Legendre transform $\mathcal{L}$ on $\operatorname{Cvx}_{0}\left(\mathbb{R}^{n}\right)$ (which seems less natural), for example

$$
\mathcal{L} \phi=\operatorname{epi}^{-1}\left(\operatorname{ref}\left(K_{\phi}^{\circ}\right)\right) .
$$

Another interesting property of the transform $\mathcal{J}$, which also gives it a geometric meaning, is the following. The transform $\mathcal{J}$, as acting on epigraphs in $\mathbb{R}^{n} \times \mathbb{R}^{+}$, is in fact induced by a point map. More precisely, if we let (for $x \in \mathbb{R}^{n}, r>0$ )

$$
F(x, r)=(x / r, 1 / r)
$$

then, defining the open epigraph of $f$ by $\operatorname{Epi}(f)=\{(x, r): f(x)<r\}$ (so that $F$ will be defined on this set) we have

$$
F(\operatorname{Epi}(f))=\{(x / r, 1 / r): f(x) \leq r\}=\{(y, s): f(y / s)<1 / s\},
$$

that is,

$$
F(\operatorname{Epi}(f))=\{(y, s): s f(y / s)<1\},
$$

which is exactly our definition of $\operatorname{Epi}(\mathcal{J} f)$. (The map $F$ is not defined, and cannot be extended, to $r=0$, which is why, if we worked with epi $(f)$, we would need to consider $F$ only on the interior, and then after applying $F$, take the closure.)

The map $F$ is a special map on $\mathbb{R}^{n} \times \mathbb{R}^{+}$, which one may call fractional-linear, and it is easily checked that it preserves all intervals, and thus as a map on sets it preserves convexity (in another terminology it is called a "permissible projective transformation"). We will discuss the family of such maps and the transforms they represent in more detail in a forthcoming paper [1] where duality and order preservation of convex sets, and convex functions defined in "windows" (that is, which lie in, or are defined on, subsets of $\mathbb{R}^{n}$ ) will be investigated.

Finally, we remark on a third way to view $\mathcal{J}$. Let $e$ stand for a unit vector in the direction of $\mathbb{R}^{+}$, orthogonal to $\mathbb{R}^{n}$ where the function is defined. We claim that

$$
\operatorname{ref}(\operatorname{epi}(\mathcal{J} f))=\left((\operatorname{epi}(f)-e)^{\circ}+e\right)^{\circ}-e .
$$


Transferring this knowledge to $\mathcal{L}$, we see a funny description for the Legendre transform on the class $\operatorname{Cvx}_{0}\left(\mathbb{R}^{n}\right)$ (the reflections in $\mathcal{A}$ and $\mathcal{J}$ cancel):

$$
\operatorname{epi}(\mathcal{L} f)=\left(\left((\operatorname{epi} f)^{\circ}+e\right)^{\circ}-e\right)^{\circ}+e .
$$

Similarly,

$$
\operatorname{epi}(\mathcal{L} f)=\left(\left((\text { epi } f-e)^{\circ}+e\right)^{\circ}-e\right)^{\circ} .
$$

By using the other interpretation for $\mathcal{J}$, we can see that defining (for $r<0$ )

$$
G(x, r)=(x / r,-1 / r)
$$

we have, for $f \in \operatorname{Cvx}_{0}\left(\mathbb{R}^{n}\right)$,

$$
\operatorname{epi}(\mathcal{L} f)=\overline{G\left((\operatorname{epi}(f))^{\circ}\right)}
$$

(here, again, we do not apply $G$ to $(x, r)$ with $r=0)$. In other words, the Legendre transform is, too, a variant of the usual duality for sets, but combined with the fractional linear map $G$.

\section{More calculus of the new transforms}

Recall the definition of the inf-convolution of two functions $\phi, \psi \in \operatorname{Cvx}\left(\mathbb{R}^{n}\right)$, given by

$$
(\phi \square \psi)(z)=\inf _{x+y=z}(\phi(x)+\psi(y)) .
$$

It is easily checked that the new function is convex as well. However, the lower-semicontinuity may be violated; to simplify notation below, we will not use a different notation for the lower-semicontinuous regularization of the above defined function, and still denote it by $\phi \square \psi$. Under this convention, it is easily verified that for $\phi, \psi \in \mathrm{Cvx}_{0}\left(\mathbb{R}^{n}\right)$, also $\phi \square \psi \in \mathrm{Cvx}_{0}\left(\mathbb{R}^{n}\right)$.

The Legendre transform exchanges the operation of inf-convolution with usual summation:

$$
\mathcal{L} \phi+\mathcal{L} \psi=\mathcal{L}(\phi \square \psi) .
$$

The operation $\square$ has a simple geometric meaning: it is the function whose epigraph corresponds to the Minkowski sum of the epigraphs of the original functions:

$$
\operatorname{epi}(\phi \square \psi)=\operatorname{epi}(\phi)+\operatorname{epi}(\psi) .
$$

Indeed,

$$
\begin{aligned}
\operatorname{epi}(\phi)+\operatorname{epi}(\psi) & =\left\{(x, y): x=x_{1}+x_{2}, y \geq \phi\left(x_{1}\right)+\psi\left(x_{2}\right)\right\} \\
& =\left\{(x, y): y \geq \inf _{x_{1}+x_{2}=x}\left(\phi\left(x_{1}\right)+\psi\left(x_{2}\right)\right)\right\}=\operatorname{epi}(\phi \square \psi) .
\end{aligned}
$$

Here one should be again careful, as the Minkowski sum of two closed sets need not be closed; thus, the following identity holds only for the regularized inf-convolution:

$$
\mathcal{L} \phi \square \mathcal{L} \psi=\mathcal{L}(\phi+\psi),
$$


and correspondingly

$$
\operatorname{epi}(\mathcal{L}(\phi+\psi))=\overline{\operatorname{epi}(\mathcal{L} \phi \square \mathcal{L} \psi)} .
$$

Since the epigraph description is one of two equally natural ways to associate a convex set of the aforementioned special form (a closed convex set which lies in the half-space $\mathbb{R}^{n} \times \mathbb{R}_{\geq 0}$ and includes the ray $\{0\} \times \mathbb{R}_{\geq 0}$ ), we can define another kind of "convolution of functions" by taking the Minkowski sum of the two sets $K_{\phi}$ and $K_{\psi}$, and letting $\phi \square \psi$ be defined by the relation

$$
K_{\phi \boxminus \psi}=\overline{K_{\phi}+K_{\psi}} .
$$

Indeed, we then actually see that

$$
\operatorname{epi}(\mathcal{J}(\phi \boxminus \psi))=K_{\phi \varpi \psi}=\overline{K_{\phi}+K_{\psi}}=\overline{\operatorname{epi}(\mathcal{J} \phi)+\operatorname{epi}(\mathcal{J} \psi)}=\operatorname{epi}(\mathcal{J} \phi \square \mathcal{J} \psi) .
$$

Thus we have the relations

$$
\mathcal{J} \phi \square \mathcal{J} \psi=\mathcal{J}(\phi \square \psi) \quad \text { and } \quad \phi \square \psi=\mathcal{J}((\mathcal{J} \phi) \square(\mathcal{J} \psi))
$$

which could also serve as a definition, and by a change of variables, implies

$$
\mathcal{A} \phi \boxminus \mathcal{A} \psi=\mathcal{A}(\phi+\psi) .
$$

By the involution property of $\mathcal{A}$, this implies that also

$$
\mathcal{A} \phi+\mathcal{A} \psi=\mathcal{A}(\phi \boxminus \psi) .
$$

We thus see that the $\mathcal{A}$ transform exchanges the operation of $\square$-convolution with usual summation, just as $\mathcal{L}$ exchanges $\square$-convolution (i.e., inf-convolution) with usual summation. We will call the operation of $\square$-convolution "geometric-inf-convolution" or "g-infconvolution" (where "g" can also stand for "gauge").

To understand better the operation of g-inf-convolution, let us compute several examples. For two closed convex sets $K_{1}$ and $K_{2}$ containing 0 ,

$$
\|\cdot\|_{K_{1}} \triangleright\|\cdot\|_{K_{2}}=\left(\mathcal{A}\|\cdot\|_{K_{1}}^{*}\right) \triangleright\left(\mathcal{A}\|\cdot\|_{K_{2}}^{*}\right)=\mathcal{A}\left(\|\cdot\|_{K_{1}}^{*}+\|\cdot\|_{K_{2}}^{*}\right)=\|\cdot\|_{K_{1}+K_{2}} .
$$

Compare this with

$$
\|\cdot\|_{K_{1}} \square\|\cdot\|_{K_{2}}=\mathcal{L}\left(1_{K_{1}^{\circ}}^{\infty}+1_{K_{2}^{\circ}}^{\infty}\right)=\mathcal{L}\left(1_{K_{1}^{\circ} \cap K_{2}^{\circ}}^{\infty}\right)=\|\cdot\|_{\left(K_{1}^{\circ} \cap K_{2}^{\circ}\right)^{\circ}}=\|\cdot\|_{\operatorname{conv}\left(K_{1} \cup K_{2}\right)} .
$$

Similarly, $1_{K_{1}}^{\infty} \square 1_{K_{2}}^{\infty}=1 \frac{\infty}{\operatorname{conv}\left(K_{1} \cup K_{2}\right)}$, which can be compared with $1_{K_{1}}^{\infty} \square 1_{K_{2}}^{\infty}=1 \frac{\infty}{K_{1}+K_{2}}$. We end this section with a list of some simple properties of $\mathcal{J}$.

1. Order preserving. Obviously, $\mathcal{J}$ is order preserving: if $f \leq g$ then $\mathcal{J} f \leq \mathcal{J} g$.

2. Involution. One may directly compute that $\mathcal{J} \mathcal{J} f=f$. It is deduced as Corollary 6 and also follows very easily from the geometric interpretation of $\mathcal{J}$ in Section 4.

We remark that the two properties listed above, in the case of $\operatorname{Cvx}\left(\mathbb{R}^{n}\right)$, characterize the à-la-identity transforms, which must be of the form $f \mapsto f \circ B$ for some $B \in \mathrm{GL}_{n}$ with $B \circ B=\mathrm{Id}$. The surprising fact is that when one restricts the class, not by much, to $\operatorname{Cvx}_{0}\left(\mathbb{R}^{n}\right)$, exactly one new transform, very different from the identity, satisfying these properties, emerges: $\mathcal{J}$. 
3. Preservation of max and min. Because $\mathcal{J}$ is order preserving and bijective it is easy to see that

$$
\mathcal{J}(\max (f, g))=\max (\mathcal{J} f, \mathcal{J} g) \quad \text { and } \quad \mathcal{J}(\hat{\min }(f, g))=\hat{\min }(\mathcal{J} f, \mathcal{J} g)
$$

where as usual $\min (f, g)$ denotes the largest function in the class which is below both $f$ and $g$. A similar statement holds for sup and inf of an arbitrarily large family of functions.

4. Homogeneity. For $a>0$,

$$
\mathcal{J}(a f)(y)=(1 / a)(\mathcal{J} f)_{a}(y), \quad \mathcal{J}\left(f_{a}\right)(y)=(\mathcal{J} f)_{a}(y) .
$$

5. Action on norms. For any norm $\|\cdot\|$, we have

$$
(\mathcal{J}\|\cdot\|)(y)=1_{K}^{\infty},
$$

where as before $K$ is the unit ball $\{x:\|x\| \leq 1\}$ and $1_{K}^{\infty}$ is the convex function attaining 0 on $K$ and $+\infty$ elsewhere. Here $\|\cdot\|$ is a generalized norm, so that for example one can check that (letting $\mathbb{R}^{+} z$ denote the ray emanating from 0 in direction $z$ )

$$
\mathcal{J}\left(1_{[0, z]}^{\infty}\right)(x)=\left\{\begin{array}{ll}
x / z, & x \in \mathbb{R}^{+} z \\
+\infty, & \text { otherwise }
\end{array} \quad\left(=\|x\|_{[0, z]}\right) .\right.
$$

6. Action on powers of norms. In fact, for every power $p \geq 1$ we have

$$
\mathcal{J}\left(\|\cdot\|^{p}\right)=\|\cdot\|^{q}
$$

where $1 / p+1 / q=1$. In particular, $\mathcal{J}\left(|\cdot|^{2} / 2\right)(y)=(1 / 2)|y|^{2}$ and $\mathcal{J}\left(\|\cdot\|^{2} / 2\right)(y)=$ $\|y\|^{2} / 2$ for any generalized norm. That is, $\mathcal{J}$ coincides with the identity transform on the subclass of 2-homogeneous functions, but for $p$-homogeneous functions $p \neq 2$ these are very different transforms.

6. Ray-wise action. This property is clear from the definition of $\mathcal{J}$, but is worth stating explicitly none the less: if for some ray $R=\mathbb{R}^{+} y$ we have $\left.f\right|_{R}=\left.g\right|_{R}$ then also $\left.\mathcal{J} f\right|_{R}=\left.\mathcal{J} g\right|_{R}$.

\section{Proof of the uniqueness theorem}

In this section we prove Theorem 2 which states that if a transform on $\mathrm{Cvx}_{0}\left(\mathbb{R}^{n}\right)$ satisfies abstract duality, it must be, up to a choice of coordinates, either $\mathcal{A}$ or $\mathcal{L}$.

We will relax the involution condition, as mentioned in Section 3 , and concentrate first on the proof of Theorem 4, that a bijective transformation on $\mathrm{Cvx}_{0}\left(\mathbb{R}^{n}\right)$ which is order reversing, and so is its inverse, must be $\mathcal{L}$ or $\mathcal{A}$ up to linear terms. Theorem 2 will follow easily from Theorem 4 A theorem completely equivalent to Theorem 4 is Theorem 5 stating that if a bijective transform on $\mathrm{Cvx}_{0}\left(\mathbb{R}^{n}\right)$ is order preserving, and so is its inverse, then it is essentially either the identity or $\mathcal{J}=\mathcal{L} \mathcal{A}$. 
Note that, since $\mathcal{J}$ is an involution, we see in particular that all bijective order preserving (in both directions) maps are essentially involutions, as are all order reversing maps. In particular, there is no order reversing map which is the "square root" of $\mathcal{J}$, and in the category of bijective maps which preserve comparability of functions, as do their inverses, there are, essentially, only four roots of identity, all of them of order 2 (we will not elaborate on this fact in this paper).

\subsection{First simple observations}

We recall the following lemma, which is standard in the framework of ordered sets, and which says that in the case of order preserving transformations, minimum is mapped to minimum and maximum to maximum. Here minimum should be understood in the sense of partial order, the largest function in the class which is less than all the functions participating in the minimum. More precisely, we let inf $\left(f_{\alpha}\right)$ stand for $\sup \left\{g: g \leq f_{\alpha} \forall \alpha\right\}$ and note that this supremum is attained and belongs to $\operatorname{Cvx}_{0}\left(\mathbb{R}^{n}\right)$.

Lemma 7. If $\mathcal{T}: \operatorname{Cvx}_{0}\left(\mathbb{R}^{n}\right) \rightarrow \operatorname{Cvx}_{0}\left(\mathbb{R}^{n}\right)$ is a bijective transformation, order preserving in both directions, that is,

1. $\phi \leq \psi$ implies $\mathcal{T} \phi \leq \mathcal{T} \psi$,

2. $\mathcal{T} \phi \leq \mathcal{T} \psi$ implies $\phi \leq \psi$,

then $\mathcal{T}\left(\sup \left(f_{\alpha}\right)\right)=\sup \left(\mathcal{T} f_{\alpha}\right)$ and $\mathcal{T}\left(\hat{\inf }\left(f_{\alpha}\right)\right)=\hat{\inf }\left(\mathcal{T} f_{\alpha}\right)$.

Lemma 7 , was proved in a more general setting for example in [5, Lemma 4].

We will later use the term positively-linearly-independent to mean $x$ and $y$ such that $x \neq t y$ for any $t \geq 0$. We use $\mathbb{R}^{+} x$ to denote the ray $\{t x: t \geq 0\}$. Also, we recall that the support of a function $f \in \operatorname{Cvx}_{0}\left(\mathbb{R}^{n}\right)$ is defined to be the set of points $x$ where $f(x) \neq \infty$, in particular 0 is always a point in the support. (Note that we do not take the closure of the set of finitely valued points, and in general this convex set need not be closed). When we say that a function is supported on a certain set, we mean that its support is included in the set (as opposed to saying that its support is a certain set, which means the support equals the set). Note that the only function in $\operatorname{Cvx}_{0}\left(\mathbb{R}^{n}\right)$ with support $\{0\}$ is $1_{\{0\}}^{\infty}$, the function which is 0 at 0 and $+\infty$ elsewhere.

We begin by an almost trivial fact, that the smallest function in the class $\operatorname{Cvx}_{0}\left(\mathbb{R}^{n}\right)$, the constant function 0 , and the largest function, $1_{\{0\}}^{\infty}$, are fixed points for $\mathcal{T}$.

Lemma 8. If $\mathcal{T}: \operatorname{Cvx}_{0}\left(\mathbb{R}^{n}\right) \rightarrow \operatorname{Cvx}_{0}\left(\mathbb{R}^{n}\right)$ is a bijective transformation, order preserving in both directions, then $\mathcal{T} 1_{\{0\}}^{\infty}=1_{\{0\}}^{\infty}$ and $\mathcal{T} 0=0$.

Proof. $1_{\{0\}}^{\infty}$ is the largest function in the class, and 0 the smallest, that is, any $f \in$ $\operatorname{Cvx}_{0}\left(\mathbb{R}^{n}\right)$ satisfies $0 \leq f \leq 1_{\{0\}}^{\infty}$. Therefore, any $g=\mathcal{T} f$ satisfies $\mathcal{T} 0 \leq g \leq \mathcal{T} 1_{\{0\}}^{\infty}$, in particular $g=0$ and $g=1_{\{0\}}^{\infty}$ (we are using the assumption that $\mathcal{T}$ is onto), which implies $\mathcal{T} 1_{\{0\}}^{\infty}=1_{\{0\}}^{\infty}$ and $\mathcal{T} 0=0$.

Remark. Although conditions 1 and 2 above are similar, they are by no means equivalent, even under the assumption of bijectivity. Indeed, one may present a bijective map $\mathcal{T}: \operatorname{Cvx}_{0}\left(\mathbb{R}^{n}\right) \rightarrow \operatorname{Cvx}_{0}\left(\mathbb{R}^{n}\right)$ which satisfies condition 1 and does not satisfy 2. An 
example is the following: for a function with bounded support, let $\mathcal{T} f=2 f$, and for a function with unbounded support, let $\mathcal{T} f=f$. Then if $f \leq g$ and both are of bounded support, or both of unbounded support, then clearly also $\mathcal{T} f \leq \mathcal{T} g$. Also, if $f \leq g$ then it cannot be that $f$ is of bounded support and $g$ is of unbounded support, so the only option left is that $f$ is of unbounded support and $g$ of bounded support, in which case $\mathcal{T} f=f \leq g \leq 2 g$. So, condition 1 holds. On the other hand, it is easy to construct two functions $f \not \leq g$ and $f \leq 2 g$ where $f$ is of unbounded support and $g$ is of bounded support (simply take $f=2 g$ on a bounded region which will be the support of $g$ ), so that condition 2 does not hold for this simple transform-which is clearly non-linear.

\subsection{Acting on rays}

We next prove a lemma which suggests that the one-dimensional case (in fact, the case of functions on $\mathbb{R}^{+}$) is almost identical to the $n$-dimensional one. Indeed, Theorem 5 will be later proved with the aid of such a restriction.

Lemma 9. If $\mathcal{T}: \operatorname{Cvx}_{0}\left(\mathbb{R}^{n}\right) \rightarrow \operatorname{Cvx}_{0}\left(\mathbb{R}^{n}\right)$ is a bijective transformation, order preserving in both directions, then there is a bijection $\Phi: S^{n-1} \rightarrow S^{n-1}$ such that any function supported on $\mathbb{R}^{+} y$ is mapped to a function supported on $\mathbb{R}^{+} z$ for $z=\Phi(y)$. Moreover, the function $1_{\mathbb{R}^{+} y}^{\infty}$ is mapped to the function $1_{\mathbb{R}^{+} z^{+}}^{\infty}$.

As a corollary such a transform must act ray-wise, namely the resulting function's value at a point $z$ depends only on the values of $f$ on the ray $\mathbb{R}^{+} x$ for $x=\Phi^{-1} z$. Indeed, the values of $\mathcal{T} f$ on $\mathbb{R}^{+} z$ are the same as the values of $\max \left(\mathcal{T} f, R_{z}\right)$ where $R_{z}$ denotes the function which is 0 on $\mathbb{R}^{+} z$ and $+\infty$ elsewhere. This maximum is the image of the function $\max \left(f, R_{y}\right)$ because $\mathcal{T} R_{y}=R_{z}$ (each being the smallest function supported on the corresponding ray). Since $\max \left(f, R_{y}\right)$ does not depend on the values $f$ attains outside $R_{y}$, our claim follows. We remark that if we were to prove the theorem directly for order reversing transformations then we would "miss" this ray-wise behavior, and get a transform $\mathcal{A}$ (or $\mathcal{L}$ ) which, when combined with $\mathcal{L}$, miraculously acts ray-wise.

Notice that for any two non-zero positively-linearly-independent vectors $x, y \in \mathbb{R}^{n}$ we can construct two functions $f_{x}$ and $f_{y}$ supported on $\mathbb{R}^{+} x$ and $\mathbb{R}^{+} y$, respectively, such that $\max \left(f_{x}, f_{y}\right)=1_{\{0\}}^{\infty}$, and thus also $\max \left(\mathcal{T} f_{x}, \mathcal{T} f_{y}\right)=1_{\{0\}}^{\infty}$, which means that they are supported on different sets. Later we will show that $\Phi$ must be linear. For now we prove Lemma 9 .

Proof of Lemma 9. For two functions $f, g$ to have $\max (f, g)=1_{\{0\}}^{\infty}$ they must be supported on two sets whose intersection equals $\{0\}$. A function with support in a line cannot be mapped to one whose support includes two positively-linearly-independent points because then $\mathcal{T}^{-1}$ would map two functions whose supports intersect at $\{0\}$ only, to functions supported on the same ray-impossible. Thus functions supported on a given ray are all mapped to functions supported on another fixed ray. From invertibility, we see that this defines a mapping $\Phi: S^{n-1} \rightarrow S^{n-1}$ which is 1-1 and onto. Moreover, the function $R_{x}$ which is constant 0 on the ray $\mathbb{R}^{+} x$ and $+\infty$ elsewhere is the smallest function supported on the ray, and hence is mapped (by surjectivity) to the smallest function supported on the ray $\mathbb{R}^{+} \Phi(x)$, which is $R_{\phi(x)}$. The proof is complete. 


\subsection{Convex functions on one ray}

So far, we see that the one-dimensional (half-line) case will determine more or less everything, up to understanding which $\Phi$ can arise in Lemma 9, and how the "free variables" allowed for each ray-transform fit together. We thus restrict our attention to dimension one (in fact, to a half-line) for a while.

In the case of all convex functions (see [4]), the functions which played a key role were the à-la-delta functions, $1_{\{u\}}^{\infty}$, assuming the value 0 at $u$ and infinity elsewhere, and the linear functions $l_{u}(x)=\langle u, x\rangle$. The first have the property that any two functions above $1_{\{u\}}^{\infty}$ are comparable, a property holding only for functions of the form $1_{\{u\}}^{\infty}+C$, and the second have the property that any two functions below $l_{u}$ are comparable, again a property holding only for functions of the form $l_{u}+c$.

Since both these classes of functions are outside $\operatorname{Cvx}_{0}\left(\mathbb{R}^{n}\right)$ (except for the special functions $1_{\{0\}}^{\infty}$ and 0 , whose behavior we already determined), we need a slightly more complicated property. However, we take advantage of considering just one dimension. Consider the following property of a function $f \in \mathrm{Cvx}_{0}\left(\mathbb{R}^{+}\right)$which we call (P): There exist no two functions $g, h \in \operatorname{Cvx}_{0}\left(\mathbb{R}^{+}\right)$such that $g \geq f$ and $h \geq f$ but $\max (g, h) \geq f$. We claim that the only two classes of functions satisfying $(\mathrm{P})$ are the following:

(a) $1_{[0, z]}^{\infty}$ which equals 0 on $[0, z]$ and $+\infty$ elsewhere.

(b) $l_{z}(t)=t z$.

Let us prove this fact.

Lemma 10. The only functions in $\mathrm{Cvx}_{0}\left(\mathbb{R}^{+}\right)$satisfying $(\mathrm{P})$ are either of the form $1_{[0, z]}^{\infty}$ for some $z \in \mathbb{R}^{+}$or of the form $l_{z}$ for some $z \in \mathbb{R}^{+}$.

Proof. It is easy to check that both families satisfy (P). To show that any function satisfying (P) must be of one of the two forms, we first show that if $f \in \operatorname{Cvx}_{0}\left(\mathbb{R}^{+}\right)$assumes some value $0<c \neq \infty$, it must be linear. Indeed, assume $f(x)=c$. Consider the function $1_{[0, x]}^{\infty}$ equal to 0 in $[0, x]$ and $+\infty$ elsewhere. Then (since $1_{[0, x]}^{\infty}(x)=0<f(x)$ ) we have $1_{[0, x]}^{\infty} \geq f$. Consider the function $L_{x}(y)=y \cdot c / x$. Then, on $[0, x]$, we must have $f \leq L_{x}$ from the convexity of $f$. In particular, since $1_{[0, x]}^{\infty} \geq f$ outside of $[0, x]$, we see that $\max \left(1_{[0, x]}^{\infty}, L_{x}\right) \geq f$, and so, by (P), $f=L_{x}$, that is, $f$ is of the form (b). The only other option is that $f$ assumes only the values 0 and $+\infty$, which implies it must be of the form (a).

Lemma 11. If $\mathcal{T}: \operatorname{Cvx}_{0}\left(\mathbb{R}^{+}\right) \rightarrow \mathrm{Cvx}_{0}\left(\mathbb{R}^{+}\right)$is a bijective transformation, order preserving in both directions, then either it maps all functions in the family (a) to functions in the family (b) and vice versa, or it maps all functions in the family (a) to functions in (a) and likewise the family (b) to itself.

Proof. First, property (P) is preserved under $\mathcal{T}$. Indeed, if there exist two functions $g, h \in$ $\operatorname{Cvx}_{0}\left(\mathbb{R}^{+}\right)$such that $g \geq \mathcal{T} f$ and $h \geq \mathcal{T} f$ but $\max (g, h) \geq \mathcal{T} f$, then the functions $\mathcal{T}^{-1} g$ and $\mathcal{T}^{-1} h$ contradict (P) for $f$. So, the family of functions satisfying (P) is mapped to the same family, and by Lemma 10 this is the family of functions of the form either (a) or (b). Since $\mathcal{T}^{-1}$ shares the same properties as $\mathcal{T}$, the map is surjective. 
Secondly, all the functions of the form (a) are comparable to one another and all functions of the form (b) are comparable to one another, but no function (a) is comparable to a function (b) (by $f$ and $g$ comparable we mean that either $f \leq g$ or $g \leq f$ ). Hence, once we know that one function of the form (a) is mapped to a function of the form (a) then all of them must be, and likewise if one function of the form (a) is mapped to a function of the form (b).

In this last lemma, a dichotomy, not apparent at first sight, appears. We have two very different possibilities, one corresponding to the identity transform (which clearly maps functions of the form (a) to functions of the same form, and likewise for (b)), and the other possibility corresponds to the transform $\mathcal{L} \mathcal{A}$, which-as can be checked-maps functions of the form (a) to functions of the form (b) and vice versa. Despite this dichotomy, in the statement of the next lemma we do not need to separate the two cases.

Define the "triangle" functions (for later use, we define them in $\mathbb{R}^{n}$ for any dimension $n$ ) for $z \in \mathbb{R}^{n}, z \neq 0$, and $c>0$ by

$$
\triangleleft_{z, c}(x)= \begin{cases}c x & \text { if } x \in[0, z], \\ +\infty & \text { otherwise. }\end{cases}
$$

Lemma 12. If $\mathcal{T}: \operatorname{Cvx}_{0}\left(\mathbb{R}^{+}\right) \rightarrow \operatorname{Cvx}_{0}\left(\mathbb{R}^{+}\right)$is a bijective transformation, order preserving in both directions, then it maps each triangle function $\triangleleft_{z, c}$ to a triangle function $\triangleleft_{z^{\prime}, c^{\prime}}\left(\right.$ with $z^{\prime}=z^{\prime}(z, c)$ and $\left.c^{\prime}=c^{\prime}(z, c)\right)$.

Proof. This follows easily from the last lemma, since a triangle is the maximum of a function of the form (a) and a function of the form (b), so that, using Lemma 7, in both cases of Lemma 11, triangles are mapped to triangles.

\subsection{Triangle functions}

Before we determine where triangles go, we show that the full form of the transform is determined by its behavior on triangles. We formulate this lemma in dimension $n$, but the same proof applies for $n=1$ and also for $\mathbb{R}^{+}$.

Lemma 13. Assume a bijective transform $\mathcal{T}: \operatorname{Cvx}_{0}\left(\mathbb{R}^{n}\right) \rightarrow \operatorname{Cvx}_{0}\left(\mathbb{R}^{n}\right)$ is order preserving in both directions, and there exist $B \in \mathrm{GL}_{n}$ and $C_{0} \in \mathbb{R}^{+}$such that for any $z$ and $c$ we have

$$
\mathcal{T}\left(\triangleleft_{z, c}\right)=\triangleleft_{B^{-1} z, C_{0} c}
$$

Then $\mathcal{T}$ is a variant of the identity transform defined by

$$
(\mathcal{T} \phi)(x)=C_{0} \phi(B x) .
$$

Similarly,

Lemma 14. Assume a bijective transform $\mathcal{T}: \mathrm{Cvx}_{0}\left(\mathbb{R}^{n}\right) \rightarrow \mathrm{Cvx}_{0}\left(\mathbb{R}^{n}\right)$ is order preserving in both directions, and there exist $B \in \mathrm{GL}_{n}$ and $C_{0} \in \mathbb{R}^{+}$such that for any $z$ and $c$ we have 


$$
\mathcal{T}\left(\triangleleft_{z, c}\right)=\triangleleft_{\frac{B^{-1} z}{|z| c}, \frac{C_{0}}{|z|}} \cdot
$$

Then $\mathcal{T}$ is a variant of the aforementioned transform $\mathcal{J}$ given by

$$
(\mathcal{T} \phi)(x)=C_{0}(\mathcal{J} \phi)(B x) .
$$

Proof of Lemma 13 Any $\phi \in \operatorname{Cvx}_{0}\left(\mathbb{R}^{n}\right)$ can be written as $\phi(x)=\left(\hat{i n f}_{y} \triangleleft_{y, \phi(y)}\right)(x)$, so that by Lemma 7 we have $(\mathcal{T} \phi)(x)=\inf _{y}\left(\mathcal{T}\left(\triangleleft_{y, \phi}(y)\right)\right)(x)$, which equals

$$
(\mathcal{T} \phi)(x)=\left(\inf _{y}\left(\triangleleft_{B^{-1}} y, C_{0}(\phi(y))\right)\right)(x)=C_{0}\left(\inf _{z}\left(\triangleleft_{z, \phi(B z)}\right)\right)(x)=C_{0} \phi(B x) .
$$

Proof of Lemma 14 Consider the transform $\mathcal{T} \mathcal{J}$, which is also order preserving in both directions. Let us show that $\mathcal{T} \mathcal{J}$ satisfies the conditions of Lemma 13 Indeed,

$$
\mathcal{T} \mathcal{J}\left(\triangleleft_{z, c}\right)=\mathcal{T}\left(\triangleleft_{\frac{z}{c|z|}, 1 /|z|}\right)=\triangleleft_{\frac{B^{-1} \frac{z}{c|z|}}{1 / c|z|}, C_{0} c}=\triangleleft_{B^{-1} z, C_{0} c}
$$

Thus, $\mathcal{T} \mathcal{J} \phi=C_{0} \phi(B x)$, and using the fact that $\mathcal{J}$ is an involution we get $(\mathcal{T} \phi)(x)=$ $C_{0}(\mathcal{J} \phi)(B x)$ as claimed.

\subsection{The mapping rule for triangles}

We start by analyzing the case where functions of the form (a) are mapped to functions of the same form, and likewise for the form (b). The second case will follow easily by application of $\mathcal{J}$. Clearly, a function $\phi: \mathbb{R}^{+} \rightarrow \mathbb{R}^{+}$for which $\mathcal{T} 1_{[0, x]}^{\infty}=1_{[0, \phi(x)]}^{\infty}$ is bijective and increasing (so it is continuous as well). Similarly for the function $c: \mathbb{R}^{+} \rightarrow$ $\mathbb{R}^{+}$for which $\mathcal{T}\left(l_{a}\right)=l_{c(a)}$.

Lemma 15. Assume a bijective transformation $\mathcal{T}: \operatorname{Cvx}_{0}\left(\mathbb{R}^{+}\right) \rightarrow \operatorname{Cvx}_{0}\left(\mathbb{R}^{+}\right)$is order preserving in both directions. Assume, further, that for some increasing bijective function $\phi: \mathbb{R}^{+} \rightarrow \mathbb{R}^{+}$we have $\mathcal{T} 1_{[0, x]}^{\infty}=1_{[0, \phi(x)]}^{\infty}$, and for another increasing bijective function $c: \mathbb{R}^{+} \rightarrow \mathbb{R}^{+}$we have $\mathcal{T} l_{a}=l_{c(a)}$. Then there exist constants $\alpha, \beta>0$ such that $\phi(x)=\alpha x$ and $c(a)=\beta a$.

Proof. By assumption and Lemma 7 the triangle $\triangleleft_{z, a}$ is mapped to $\triangleleft_{\phi(z), c(a)}$. Let $0<$ $t<1$ and consider the function $g=\inf \left(1_{[0, t z]}^{\infty}, l_{a /(1-t)}\right)$. Then $g \leq \triangleleft_{z, a}$, and their graphs have a common point $(z, a z)$, so that $g\left\lfloor\triangleleft_{z, a^{\prime}}\right.$ for any $a^{\prime}<a$ (or, similarly, $g \not \leq \triangleleft_{z^{\prime}, a}$ for any $z^{\prime}>z$ ).

The function $g$ is mapped to $\hat{\inf }\left(1_{[0, \phi(t z)]}^{\infty}, l_{c(a /(1-t))}\right)$, which thus must be smaller than $\triangleleft_{\phi(z), c(a)}$. In particular, we see that

$$
(\phi(z)-\phi(t z)) c(a /(1-t)) \leq \phi(z) c(a) .
$$

Further, we claim that there must be equality in this inequality, since if we replace $c(a)$ on the right hand side by any $c<c(a)$, the opposite inequality holds. 
We get

$$
1-\frac{c(a)}{c(a /(1-t))}=\frac{\phi(t z)}{\phi(z)}
$$

In particular, the ratio $\phi(t z) / \phi(z)$ does not depend on $z$, so it is the same as $\phi(t) / \phi(1)$, so that we may write

$$
\phi(t z)=\phi(z) \frac{\phi(t)}{\phi(1)} .
$$

Note that, formally, we have proved (4) for $t<1$. However, for $t>1$ we let $z^{\prime}=t z$ so that

$$
\frac{\phi(t z)}{\phi(z)}=\frac{1}{\frac{\phi\left(z^{\prime} / t\right)}{\phi\left(z^{\prime}\right)}}=\frac{1}{\frac{\phi(1 / t)}{\phi(1)}}=\frac{1}{\frac{\phi(1)}{\phi(t)}}=\frac{\phi(t)}{\phi(1)},
$$

where for the last but one equality we have used (5) with $1 / t$ instead of $t$ and $t$ instead of $z$ (which we may, since $1 / t>1$ ).

Equation (5), valid for all $t, z>0$, together with the continuity of $\phi$ implies that $\phi$ is of the form

for some fixed $\alpha>0$ and $\gamma$.

$$
\phi(z)=\alpha z^{\gamma}
$$

The same is true for $c(a)$ : the ratio $\frac{c(a)}{c(a /(1-t))}$ does not depend on $a$, so it is the same as $\frac{c(1)}{c(1 /(1-t))}$, so that we may write

$$
c(s a)=c(s) \frac{c(a)}{c(1)},
$$

which applies also for $s>1$ by the same argument given for (5) above, and it implies that for some $\beta>0$ and $\gamma^{\prime}$ we have

$$
c(a)=\beta a^{\gamma^{\prime}} .
$$

Next, by the identity (4) above, for all $t>0$,

$$
1-(1-t)^{\gamma^{\prime}}=t^{\gamma}
$$

which can hold for all $t$ only if $\gamma=\gamma^{\prime}=1$.

Next we analyze the case where functions of the form (a) are mapped to functions of the form (b), and vice versa. Luckily, we know one transform which indeed works this way, and we may use it (and the fact that it is an involution), together with the previous lemma, to give a simple proof of the following lemma.

Lemma 16. Assume a bijective transformation $\mathcal{T}: \mathrm{Cvx}_{0}\left(\mathbb{R}^{+}\right) \rightarrow \mathrm{Cvx}_{0}\left(\mathbb{R}^{+}\right)$is order preserving in both directions. Assume, further, that for some increasing bijective function $c: \mathbb{R}^{+} \rightarrow \mathbb{R}^{+}$we have $\mathcal{T} 1_{[0, x]}^{\infty}=l_{c(x)}$, and for another increasing bijective function $\phi: \mathbb{R}^{+} \rightarrow \mathbb{R}^{+}$we have $\mathcal{T} l_{a}=1_{[0, \phi(a)]}^{\infty}$. Then there exist constants $\alpha, \beta>0$ such that $\phi(a)=\beta / a$ and $c(x)=\alpha / x$.

Proof. Apply $\mathcal{J}$, so that $\mathcal{J} \mathcal{T} 1_{[0, x]}^{\infty}=1_{[0,1 / c(x)]}^{\infty}$ and $\mathcal{J} \mathcal{T} l_{a}=l_{1 / \phi(a)}$. (Note that we are using the properties of the transform $\mathcal{J}$, which were studied in Section 5 , specifically property 3: homogeneity of degree -1 ). We may thus apply, to the new transform $\mathcal{J} \mathcal{T}$, Lemma 15, and we see that $c(x)=\alpha / x$ and $\phi(a)=\beta / a$. 


\subsection{The theorem on a ray}

Let us regroup what we have shown so far. First, we have all the ingredients for the following one-dimensional theorem.

Theorem 17. If $\mathcal{T}: \operatorname{Cvx}_{0}\left(\mathbb{R}^{+}\right) \rightarrow \operatorname{Cvx}_{0}\left(\mathbb{R}^{+}\right)$is a bijective transformation, order preserving in both directions, then there exist constants $\alpha, \beta>0$ such that either (⿳亠口冋-laidentity) for every $\phi \in \mathrm{Cvx}_{0}\left(\mathbb{R}^{+}\right)$,

$$
(\mathcal{T} \phi)(x)=\beta \phi(x / \alpha)
$$

or (à-la-J $)$, for every $\phi \in \operatorname{Cvx}_{0}\left(\mathbb{R}^{+}\right)$,

$$
(\mathcal{T} \phi)(x)=\beta(\mathcal{J} \phi)(x / \alpha) .
$$

Proof. Given such a $\mathcal{T}$, we use Lemma 11 to deduce that there are two options: either all functions of the form (a) are mapped to functions in the family (b), and likewise (b) to (a), or $\mathcal{T}$ maps all functions in (a) to functions in (a), and likewise the family (b) to itself. Let us start with the second option (which we call à-la-identity). We then infer, by Lemma 15 and the discussion before it, that there exist constants $\alpha, \beta>0$ such that $\mathcal{T} 1_{[0, x]}^{\infty}=1_{[0, \alpha x]}^{\infty}$ and $\mathcal{T} l_{a}=l_{\beta a}$. Using Lemma 7 we see that $\mathcal{T}\left(\triangleleft_{z, a}\right)=\triangleleft_{\alpha z, \beta a}$ for every $z$ and $a$ in $\mathbb{R}^{+}$. We then use Lemma 13 to get $(\mathcal{T} \phi)(x)=\beta \phi(x / \alpha)$. The other case is when the families (a) and (b) are interchanged. Lemma 16 implies that $\mathcal{T} 1_{[0, x]}^{\infty}=l_{\alpha / x}$ and $\mathcal{T} l_{a}=1_{[0, \beta / a]}^{\infty}$. Using Lemma 7 we see that $\mathcal{T}\left(\triangleleft_{z, a}\right)=\triangleleft_{\beta / a, \alpha / z}$, which by Lemma 14 implies that $(\mathcal{T} \phi)(x)=\alpha(\mathcal{J} \phi)(x / \beta)$.

\subsection{Completing the proof in $n$ dimensions}

We turn next to the higher dimensional case, namely when $\mathcal{T}: \operatorname{Cvx}_{0}\left(\mathbb{R}^{n}\right) \rightarrow \operatorname{Cvx}_{0}\left(\mathbb{R}^{n}\right)$ and $n \geq 2$. We know, by Lemma 9 , that there is some (bijective) $\Phi: S^{n-1} \rightarrow S^{n-1}$ such that any function supported on $\mathbb{R}^{+} y$ is mapped to a function supported on $\mathbb{R}^{+} z$ for $z=\Phi(y)$. Moreover, the function $1_{\mathbb{R}^{+} y}^{\infty}$ is mapped to the function $1_{\mathbb{R}^{+} z}^{\infty}$. Thus, for each $y \in S^{n-1}$ we actually have a mapping from $\operatorname{Cvx}_{0}\left(\mathbb{R}^{+} y\right)$ (which is isometric, say via $I_{1}$, to $\operatorname{Cvx}_{0}\left(\mathbb{R}^{+}\right)$) to $\operatorname{Cvx}_{0}\left(\mathbb{R}^{+} \Phi(y)\right.$ ) (again isometric, say via $I_{2}$, to $\operatorname{Cvx}_{0}\left(\mathbb{R}^{+}\right)$). So we may apply Theorem 17 to the transform $I_{2} \circ \mathcal{T} \circ I_{1}^{-1}$, and deduce that there are constants $\beta_{y}$ and $\alpha_{y}$ for which the conclusion of Theorem 17 holds.

Our goal now is first to show that the same option of Theorem 17] holds for all $y \in$ $S^{n-1}$ (that is, the transform is either à-la-identity for all $y$ 's, or à-la- $\mathcal{J}$ for all $y$ 's), and secondly that we can specify which $\alpha_{y}, \beta_{y}$ and $\Phi$ can occur.

Let us remark that since $\Phi$ clearly sends cones to cones (that is, spherically-convex sets on the sphere to spherically-convex sets on the sphere), and also preserves, on the sphere, intersections and convex hulls of unions of cones (by Lemma 7; simply take functions which are 0 on the cone spanned by the set; the intersection is then given by the supremum, and the convex hull by inf). Thus, in dimension higher than 2 , we can use the theorem of Schneider [17] to deduce that $\Phi$ must be linear (as acting on cones). Instead, 
we give an independent proof which works also for lower dimensions, and to this end we will invoke the full structure of our set $\operatorname{Cvx}_{0}\left(\mathbb{R}^{n}\right)$.

For the first step, we associate to each $y$ the number $j(y)$ which is 1 if $I_{2} \circ \mathcal{T} \circ I_{1}^{-1}$ is à-la- $\mathcal{J}$ (that is, $I_{2} \circ \mathcal{T} \circ I_{1}^{-1} \phi=\alpha_{y}(\mathcal{J} \phi)\left(x / \beta_{y}\right)$ and 0 if it is à-la-identity (i.e., $\left.I_{2} \circ \mathcal{T} \circ I_{1}^{-1} \phi=\beta_{y} \phi\left(x / \alpha_{y}\right)\right)$. We claim $j$ is constant, either always 0 or always 1 .

Indeed, consider the function $1_{B}^{\infty}$, which is 0 on the unit ball $\{y:|y| \leq 1\}$ and $+\infty$ elsewhere. It is mapped to a function which is, in direction $\Phi(y)$, linear if $j(y)=1$ and of the form $1_{\left[0, \alpha_{y} \Phi(y)\right]}^{\infty}$ if $j(y)=0$. Note that the function cannot be identically 0 on a ray, nor $1_{\{0\}}^{\infty}$ on a ray, since we already know that the restricted transforms map these functions to themselves and are injective. We claim that no convex function can be, on rays, only of the forms either linear (non-zero) or $1_{[0, y]}^{\infty}$, and attain both these forms.

Indeed, it is easy to check that if in two directions the function is linear, then on any direction which is a convex combination of the two, the function cannot have compact support (as the support is convex). Similarly, if in two directions the function is of the form $1_{[0, y]}^{\infty}$ and $1_{[0, z]}^{\infty}$, then it cannot be linear on a direction which is a convex combination of the two (since it must be 0 on $\operatorname{conv}(0, y, z)$ ). Thus, the collection of rays for which $j(y)=0$ is convex, as is the subset of rays for which $j(y)=1$. Since this splits all rays into two disjoint subsets, we see that, unless one of them is empty, each is a half-space through 0 . Let us denote by $H$ the half-space of rays where the function is linear. We will get a contradiction by considering a ray (say, in direction $y_{0}$ ) which is on the boundary of $H$.

Indeed, if $j\left(y_{0}\right)=0$, then the support of the function must include the half-space $H$, but does not include the whole ray in direction $y_{0}$. However, a convex set which includes an open half-space and at least one extra point outside its closure, must include the closure itself. Since we know that the support includes points outside the closure (because on no ray is the function equal to $\left.1_{\{0\}}^{\infty}\right)$, this situation is impossible, and we must have $j\left(y_{0}\right)=1$. However, this too leads to a contradiction, because the function then has finite and non-zero values on the whole ray in direction $y_{0}$. Take some line in $\mathbb{R}^{n}$ which intersects the ray transversally (not at 0 ), and restrict the function to this line. It clearly should remain convex. However, on the half-line which lies in $H$, the function assumes finite and non-zero values, and also at the crossing point. On the half-line which lies in the complementing half-space, the function assumes only values 0 and $+\infty$. If it assumes the value 0 on this half-space, then it cannot be convex (it has no way to "jump", convexly, from the value 0 to a finite non-zero value). So we see that it must attain only the value $+\infty$. But this cannot hold true for all lines crossing the ray, as the function is not constant $+\infty$ on the complementary half-space. This completes the proof that $j$ is a constant function.

Let us next deal with the case that $j \equiv 0$. We define $\varphi: \mathbb{R}^{n} \rightarrow \mathbb{R}^{n}$ by $\mathcal{T} 1_{[0, x]}^{\infty}=$ $1_{[0, \varphi(x)]}^{\infty}$. (Clearly the "projective" part of $\varphi$ is $\Phi$.) The function $\varphi$ is clearly bijective. From the properties of $\mathcal{T}$ it follows that $\mathcal{T} 1_{K}^{\infty}=1_{\varphi(K)}^{\infty}$ for a closed convex set $0 \in K \subset \mathbb{R}^{n}$. Therefore, $\varphi: \mathbb{R}^{n} \rightarrow \mathbb{R}^{n}$ preserves convexity and is thus linear, by well known results (we have used here that $n \geq 2$ ). Denote by $B \in \mathrm{GL}_{n}$ the inducing linear map, $\varphi(x)=B x$.

Next, we denote by $a(x, c)$ the height of the triangle which is the image of the triangle with base $[0, x]$ and height $c$. 
Since the largest triangle with base $\left[0, \lambda x+(1-\lambda) x^{\prime}\right]$ which is smaller than the infimum of the triangle with base $[0, x]$ and height $c$ and the triangle with base $\left[0, x^{\prime}\right]$ and height $c^{\prime}$ has height $\lambda c+(1-\lambda) c^{\prime}$, and these are mapped to new triangles of bases $[0, B x],\left[0, B x^{\prime}\right]$ and $\left[0, \lambda B x+(1-\lambda) B x^{\prime}\right]$ with heights $a(x, c), a\left(x^{\prime}, c^{\prime}\right)$ and $a\left(\lambda x+(1-\lambda) x^{\prime}, \lambda c+(1-\lambda) c^{\prime}\right)$ respectively, we get $a\left(\lambda(x, c)+(1-\lambda)\left(x^{\prime}, c^{\prime}\right)\right)=$ $\lambda a(x, c)+(1-\lambda) a\left(x^{\prime}, c^{\prime}\right)$ so that $a: \mathbb{R}^{n+1} \rightarrow \mathbb{R}^{n+1}$ is linear, that is, $a(x, c)=$ $\left\langle x, y_{0}\right\rangle+\beta_{0} c$. However, since $a(x, 0)=0$ for all $x$, we get $a(x, c)=\beta_{0} c$.

To sum up, we now know that a function $f$ which is a triangle is mapped to $\beta_{0} f \circ B$, and by Lemma 13 once we know this for triangles, we know it for all functions.

Finally, to deal with the case that $j \equiv 1$, we look at $\mathcal{T}^{\prime}=\mathcal{J} \circ \mathcal{T}$. Then for the new transform all the properties hold, and $j \equiv 0$ so that by the reasoning above $\mathcal{T}^{\prime} f=\beta_{0} f \circ B$ and so $\mathcal{T} f=\mathcal{J}\left(\beta_{0} f \circ B\right)=\left(1 / \beta_{0}\right) \mathcal{J}(f \circ B)$ as claimed. This completes the proof of Theorem 5

Remark. The proof of Theorem 3 is a modification of the proof of Theorem 17 . Indeed, we first need to show that the two subclasses of $\operatorname{Cvx}_{0}(\mathbb{R})$ consisting of functions which are identically 0 on $\mathbb{R}^{+}$(which by abuse of notation we denote $\operatorname{Cvx}_{0}\left(\mathbb{R}^{+}\right)$) and those which are identically 0 on $\mathbb{R}^{-}$(which we denote $\operatorname{Cvx}_{0}\left(\mathbb{R}^{-}\right)$) have the property that either $\mathcal{T}\left(\operatorname{Cvx}_{0}\left(\mathbb{R}^{-}\right)\right)=\operatorname{Cvx}_{0}\left(\mathbb{R}^{-}\right)$and $\mathcal{T}\left(\operatorname{Cvx}_{0}\left(\mathbb{R}^{+}\right)\right)=\operatorname{Cvx}_{0}\left(\mathbb{R}^{+}\right)$, or $\mathcal{T}\left(\operatorname{Cvx}_{0}\left(\mathbb{R}^{-}\right)\right)=$ $\operatorname{Cvx}_{0}\left(\mathbb{R}^{+}\right)$and $\mathcal{T}\left(\operatorname{Cvx}_{0}\left(\mathbb{R}^{+}\right)\right)=\operatorname{Cvx}_{0}\left(\mathbb{R}^{-}\right)$, and each of the restrictions of $\mathcal{T}$ must be bijective. After this is done, all we need is to apply Theorem 17 To show the former, we fix $f \in \operatorname{Cvx}_{0}\left(\mathbb{R}^{+}\right)$and $g \in \operatorname{Cvx}_{0}\left(\mathbb{R}^{-}\right)$; then $\inf (f, g)=0$ and so $\inf (\mathcal{T} f, \mathcal{T} g)=0$. It is not hard to show that this means that one of $\mathcal{T} f, \mathcal{T} g$ must be 0 on $\mathbb{R}^{+}$and the other on $\mathbb{R}^{-}$. To show bijectivity, do the same for $\mathcal{T}^{-1}$.

\section{Log-concave functions}

Denote by $\operatorname{LC}\left(\mathbb{R}^{n}\right)$ (for "log-concave") the class of upper-semicontinuous non-negative functions $f: \mathbb{R}^{n} \rightarrow[0, \infty)$ whose logarithm is concave (with $\log (0)=-\infty$ of course). That is,

$$
\operatorname{LC}\left(\mathbb{R}^{n}\right)=\left\{e^{-\phi}: \phi \in \operatorname{Cvx}\left(\mathbb{R}^{n}\right)\right\} .
$$

The class of log-concave functions, defined above, is studied in probability and in convex geometry (see [6], [7] and for more recent developments e.g. [13], [12], [14]). Duality for this class was first studied in a joint paper of the authors and B. Klartag [2]. Since there is a one-to-one correspondence between $\operatorname{LC}\left(\mathbb{R}^{n}\right)$ and $\operatorname{Cvx}\left(\mathbb{R}^{n}\right)$, given by $f \mapsto$ $-\log (f)$, and this correspondence is order reversing, we see that any duality transform on $\operatorname{LC}\left(\mathbb{R}^{n}\right)$ defines a duality transform on $\operatorname{Cvx}\left(\mathbb{R}^{n}\right)$ and vice versa.

Indeed, one may translate theorems from one "language" to the other. The reason for doing this is that the theorems are sometimes more cleanly presented in the language of convex functions, but on the other hand log-concave functions are understood to be, in the theory of asymptotic geometric analysis, the "right" generalization of convex bodies. Logconcave functions have been investigated intensively by many authors, who found that the intuition coming from the study of convex bodies enables one to formulate functional inequalities which turn out to be of independent interest. Also, the functional inequalities 
can sometimes be applied to functions related to a convex body, and yield strong inequalities for convex bodies. This scheme was pursued for example in [12].

A measure $\mu$ on $\mathbb{R}^{n}$ is called log-concave if for any measurable $A, B \subset \mathbb{R}^{n}$ and any parameter $0<\lambda<1$,

$$
\mu(\lambda A+(1-\lambda) B) \geq \mu(A)^{\lambda} \mu(B)^{1-\lambda},
$$

where $A+B=\{a+b: a \in A, b \in B\}$ is the Minkowski sum of $A$ and $B$ and $\lambda A=\{\lambda a: a \in A\}$ is the $\lambda$-homothety of $A$. The first example of a log-concave measure is the standard Lebesgue measure $\operatorname{Vol}_{n}$ on $\mathbb{R}^{n}$. The log-concavity of the Lebesgue measure follows from the Brunn-Minkowski inequality. Similarly, a uniform measure on a convex body is log-concave. More examples of log-concave measures stem from Brunn's concavity principle [9]. This principle states that any lower dimensional marginal of a uniform measure on a convex body is a log-concave measure. Moreover, marginals of uniform measures on convex bodies are essentially the only source of log-concave measures, as these marginals form a dense subset in the class of all log-concave measures.

The notion of a log-concave measure, and that of a log-concave function, are closely related, as was shown in [7]: a measure $\mu$ on $\mathbb{R}^{n}$ whose support (here we mean the usual support of a measure) is not contained in any affine hyperplane is log-concave if and only if it is absolutely continuous with respect to the Lebesgue measure, and its density is a log-concave function. Therefore the standard Gaussian measure on $\mathbb{R}^{n}$ with density $(\sqrt{2 \pi})^{-n / 2} \exp \left\{-|x|^{2} / 2\right\}$ is a log-concave measure, where $|\cdot|$ is the standard euclidean norm in $\mathbb{R}^{n}$.

In generalizing the theory of convex bodies to log-concave functions, one of the first tasks was to understand what is the correct definition of the dual of a function. A hint was given by the fact that log-concave functions are essentially marginals of convex bodies, and we may find a way to induce the notion of duality from convex bodies to their marginals, the log-concave measures. This idea led to the following definition:

$$
f^{\circ}(x)=\inf _{y \in \mathbb{R}^{n}} e^{-\langle x, y\rangle} / f(y) .
$$

Or, equivalently, $-\log f^{\circ}=\mathcal{L}(-\log f)$. As explained earlier, this is the only abstract duality on $\operatorname{LC}\left(\mathbb{R}^{n}\right)$, up to linear changes.

Denote by $\operatorname{LC}_{\mathrm{g}}\left(\mathbb{R}^{n}\right)$ the class of upper-semicontinuous non-negative functions $f$ : $\mathbb{R}^{n} \rightarrow[0,1]$ whose logarithm is concave, and with $f(0)=1$. That is,

$$
\operatorname{LC}_{\mathrm{g}}\left(\mathbb{R}^{n}\right)=\left\{e^{-\phi}: \phi \in \operatorname{Cvx}_{0}\left(\mathbb{R}^{n}\right)\right\}
$$

The name we suggest for this class is "geometric log-concave functions".

The class of convex bodies (or generalized bodies) can be embedded into $\operatorname{LC}\left(\mathbb{R}^{n}\right)$ in various ways, for instance, letting $1_{K}$ stand for the usual indicator function (equaling 1 on $K$ and 0 elsewhere), we can have

$$
K \mapsto 1_{K}, \quad \text { or } \quad K \mapsto f(x)=e^{-\|x\|_{K}}, \quad \text { or } \quad K \mapsto f(x)=e^{-\|x\|_{K}^{2} / 2},
$$


and many other possibilities, of which the above three are the most common (and the first, probably, most intuitive). Each such presentation has its advantages, but notice that all three mentioned above give in fact a function in $\mathrm{LC}_{\mathrm{g}}\left(\mathbb{R}^{n}\right)$. That is, at least from the point of view of log-concave functions as a generalization of convex bodies, the "normalization" condition of values between 0 and 1 , with maximum 1 attained at 0 , is very natural.

Let us define a new duality transform on the class $\operatorname{Cvx}_{\mathrm{g}}\left(\mathbb{R}^{n}\right)$. The transform $f \mapsto f^{\odot}$ is given by

$$
f^{\odot}=e^{-\mathcal{A}(-\log f)} .
$$

It is clearly, by the properties of $\mathcal{A}$, a duality transform on this class. Let us list a few simple properties (additional to the obvious duality properties, that it is order reversing, and involutive):

1. $1_{K}^{\odot}=1_{K^{\circ}}$.

This shows that our new duality transform is an extension of the standard geometric duality for convex sets, and for this reason we call it "geometric duality" (for log-concave functions). Note that the previous duality, introduced in [2], gave $\left(1_{K}\right)^{\circ}=e^{-\|x\|_{K^{\circ}}}$, and in [4] we showed that it is the only possibility if one considers all of $\operatorname{LC}\left(\mathbb{R}^{n}\right)$ : there is no duality on this class which would satisfy property 1 above. However, we see now that for $\mathrm{LC}_{\mathrm{g}}\left(\mathbb{R}^{n}\right)$ there is a duality, geometric duality, which extends the duality of convex sets. Moreover, it is unique. A few more properties of geometric duality:

2. $\left(e^{-\|\cdot\|}\right)^{\odot}=e^{-\|\cdot\|^{*}}$.

3. $\left(e^{-\|\cdot\|^{p}}\right)^{\odot}=e^{-c_{p}\left(\|\cdot\|^{*}\right)^{p}}$.

4. $\left(f_{a}\right)^{\odot}=\left(e^{-\phi_{a}}\right)^{\odot}=e^{-\mathcal{A}\left(\phi_{a}\right)}=e^{-(\mathcal{A} \phi)_{1 / a}}=\left(f^{\odot}\right)_{1 / a}$.

5. $\left(f^{a}\right)^{\odot}=\left(e^{-a \phi}\right)^{\odot}=e^{-\mathcal{A}(a \phi)}=e^{-(\mathcal{A} \phi) / a}=\left(f^{\odot}\right)^{1 / a}$.

6. $(f \cdot g)^{\odot}=\left(e^{-(\phi+\psi)}\right)^{\odot}=e^{-\mathcal{A}(\phi+\psi)}=e^{-(\mathcal{A} \phi \boxminus \mathcal{A} \psi)}$.

\section{An example of a class with no duality}

Let us point out a simple example of a relatively natural class of functions for which there is no abstract duality.

The class, which we can denote $\operatorname{Cvx}_{+}\left(\mathbb{R}^{n}\right)$, although we will not use it outside this section, is the class of all non-negative convex lower-semicontinuous functions; in other words, $\left\{f \in \operatorname{Cvx}\left(\mathbb{R}^{n}\right): f \geq 0\right\}$.

Theorem 18. There does not exist a bijective $\mathcal{T}: \mathrm{Cvx}_{+}\left(\mathbb{R}^{n}\right) \rightarrow \mathrm{Cvx}_{+}\left(\mathbb{R}^{n}\right)$ such that

$$
f \leq g \Leftrightarrow \mathcal{T} f \geq \mathcal{T} g .
$$

Proof. Consider the element $D_{x}$ which equals 0 at $x$ and $+\infty$ elsewhere. It has the property that any two functions greater than it are comparable, that is, $f \geq D_{x}$ and $g \geq D_{x}$ implies that either $f \geq g$ or $g \geq f$ (since both must be of the form $c+D_{x}$ ). Therefore, by bijectivity and order reversal, the function $\mathcal{T} D_{x}$ must have the property that any two 
functions smaller than it (and in the class) are comparable. However, for a positive convex function which is non-constant, we can always find two positive convex functions smaller than it which are not comparable. Indeed, take some supporting hyperplane $\langle u, \cdot\rangle+c$ (non-horizontal) of the function; then the function is greater than $h=\max (\langle u, \cdot\rangle+c, 0)$ and so it is enough to find two non-comparable functions below $h$. These can be taken to be $h_{1}=\max (\langle u, \cdot\rangle+c-1,0)$ and $h_{2}=h / 2=\max (\langle u / 2, \cdot\rangle+c / 2,0)$.

Thus, the only function in $\mathrm{Cvx}_{+}\left(\mathbb{R}^{n}\right)$ such that any two functions below it are comparable is a constant function. We infer that each $D_{x}$ is mapped to a constant function. But this is impossible, since constant functions are all comparable, and $D_{x}$ and $D_{y}$ are not comparable when $x \neq y$.

An interesting question is to determine abstract conditions for the existence (at all) of a duality transform on a class of functions (or a general partially ordered set). Or, even better, to find how many (up to isomorphism) duality relations there exist for a given class. This number is an invariant (under order-homomorphisms) of the class, and would give a classification of classes.

\section{Proper convex functions}

In this section we discuss one more class, which is again natural for geometric purposes, and which we call "proper geometric convex functions". This class consists of all functions in $\operatorname{Cvx}_{0}\left(\mathbb{R}^{n}\right)$ which are 0 only at 0 , and which do not assume the value $+\infty$; that is, convex functions $f: \mathbb{R}^{n} \rightarrow[0, \infty)$ with $f(x)=0 \Leftrightarrow x=0$. We denote this class by $\operatorname{Cvx}_{\mathrm{p}}\left(\mathbb{R}^{n}\right)$ (the letter "p" stands for "proper" geometric convex functions). It is easy to check that it is an invariant subclass for $\mathcal{A}$, so $\mathcal{A}$ acts as abstract duality on this class. Also, notice that it is not an invariant subclass for $\mathcal{L}$, since $\mathcal{L}$ sends some functions with only finite values to functions that take the value $+\infty$. We restrict to the case of the domain being $\mathbb{R}^{+}$, where such functions are sometimes called Young functions.

Not surprisingly, it turns out that for this class, up to linear terms, $\mathcal{A}$ is the unique duality transform.

Theorem 19. Let $\mathcal{T}: \operatorname{Cvx}_{p}\left(\mathbb{R}^{+}\right) \rightarrow \operatorname{Cvx}_{p}\left(\mathbb{R}^{+}\right)$be an order reversing involution, that is, for all $\phi, \psi \in \operatorname{Cvx}_{p}\left(\mathbb{R}^{+}\right)$,

- $\mathcal{T} \mathcal{T} \phi=\phi$,

- $\phi \leq \psi$ implies $\mathcal{T} \phi \geq \mathcal{T} \psi$.

Then there exist constants $C_{0}, b \in \mathbb{R}$ such that $(\mathcal{T} \phi)(x)=C_{0}(\mathcal{A} \phi)(b x)$.

The proof is rather similar to other proofs given in this paper, and is therefore omitted.

\section{Applications of the uniqueness result}

In this section we show how two central operations of classical convexity, the support map and the Minkowski map, which are very geometric constructions, may be uniquely defined in the language of "order preserving" and "order reversing" maps. Through this understanding they can be extended to the functional class $\operatorname{LC}\left(\mathbb{R}^{n}\right)$ (or its subclass $\operatorname{LC}_{\mathrm{g}}\left(\mathbb{R}^{n}\right)$ ) 
in a natural way. It has been realized recently that the extension of geometric notions and results from convex bodies to log-concave measures is an important goal in asymptotic geometric analysis and it is now called "geometrization of probability" (see for example the survey [14] and the references therein), because it allows extending to the probability setting some important geometric inequalities.

\subsection{The Minkowski mapping: classical}

Let us denote by $\mathcal{H}^{n}$ the space of positively-homogeneous convex functions $h: \mathbb{R}^{n} \rightarrow$ $\mathbb{R}_{\geq 0} \cup\{+\infty\}$, and by $\mathcal{H}_{(0)}^{n}$ the subclass of functions in $\mathcal{H}^{n}$ which do not assume the value $+\infty$ and are equal to 0 only at 0 . (The notation is derived from the corresponding notation for convex bodies: the closed convex set $\{x: h(x) \leq 1\}$ belongs to $\mathcal{K}^{n}$, the closed convex sets including 0 , and to $\mathcal{K}_{(0)}^{n}$, the compact convex sets with 0 in the interior, respectively.)

The Minkowski operator $M: \mathcal{K}^{n} \rightarrow \mathcal{H}^{n}$ maps a closed convex set which includes 0 to a generalized norm whose unit ball is this set, and is a bijection. Its restriction to $\mathcal{K}_{(0)}^{n}$ is onto $\mathcal{H}_{(0)}^{n}$. More precisely, it maps $K$ to the positively-homogeneous function $M(K)=\|\cdot\|_{K}$ given by

$$
M(K)(x)=\|x\|_{K}=\inf \{r>0: x / r \in K\} .
$$

It is clear that the Minkowski map is an order reversing transformation, mapping $K_{1} \subset K_{2}$ to $M\left(K_{2}\right) \leq M\left(K_{1}\right)$ (with respect to the partial order of pointwise inequality on functions).

\subsection{The support map: classical}

Similarly, it is classical and well known that to a convex body $K \in \mathcal{K}^{n}$ there corresponds a convex positively-homogeneous function $h_{K}:=\|\cdot\|_{K}^{*}$ which is its support function, given also by

$$
h_{K}(u)=\sup \{\langle x, u\rangle: x \in K\} .
$$

We denote $S(K)=h_{K}$ so that $S: \mathcal{K}^{n} \rightarrow \mathcal{H}^{n}$ is a bijection, as is $S: \mathcal{K}_{(0)}^{n} \rightarrow \mathcal{H}_{(0)}^{n}$. It is easy to see that $S$ is an order preserving transformation (with respect to the partial order of inclusion for sets and the partial order of pointwise inequality on functions).

We have of course $M(K)=S\left(K^{\circ}\right)$ and vice versa.

\subsection{Uniqueness}

Both $S$ and $M$ are (essentially) uniquely defined in the language of inequalities. This is just a restatement of a theorem from [8] (for the case of $\mathcal{K}_{(0)}^{n}$ ) and a theorem from [5] (for the case of $\mathcal{K}^{n}$ ). Recall that "essentially" only means that we allow different choices of an inner product in the linear space, and some other numerical normalization constants.

Theorem 20 (following from Böröczky-Schneider, [8]). Any bijective mapping $\mathcal{T}$ : $\mathcal{K}_{(0)}^{n} \rightarrow \mathcal{H}_{(0)}^{n}$ which preserves the (partial) order is, up to a linear change, the support map $S$ defined above. Any mapping $\mathcal{T}: \mathcal{K}_{(0)}^{n} \rightarrow \mathcal{H}_{(0)}^{n}$ which reverses the (partial) order is, up to a linear change, the Minkowski map $M$ defined above. 
Theorem 21 (following from [5]). Any bijective mapping $\mathcal{T}: \mathcal{K}^{n} \rightarrow \mathcal{H}^{n}$ which preserves the (partial) order is, up to a linear change, the support map $S$ defined above. Any mapping $\mathcal{T}: \mathcal{K}^{n} \rightarrow \mathcal{H}^{n}$ which reverses the (partial) order is, up to a linear change, the Minkowski map $M$ defined above.

Indeed, to see this simply consider the mapping $\mathcal{T}^{\prime} K=S^{-1}(\mathcal{T} K)$, which is order preserving if $\mathcal{T}$ is order preserving, or the mapping $\mathcal{T}^{\prime} K=M^{-1}(\mathcal{T} K)$, which is order preserving if $\mathcal{T}$ is order reversing, and then use the corresponding theorems which imply that in these cases $\mathcal{T}^{\prime}$ must be essentially the identity mapping of convex bodies (more precisely, there is some $B \in \mathrm{GL}_{n}$ such that $\mathcal{T}^{\prime} K=B K$ ).

\subsection{Extending the maps to log-concave functions}

Next, one may extend the operation of support function from the class $\mathcal{K}^{n}$ to the class $\operatorname{LC}\left(\mathbb{R}^{n}\right)$ (where the imbedding $\mathcal{K}^{n} \subset \operatorname{LC}\left(\mathbb{R}^{n}\right)$ is simply $K \mapsto 1_{K}$ ). The extension is given by

$$
S(f)=\mathcal{L}(-\log f) .
$$

Again this is an essentially unique order preserving mapping, this time between $\operatorname{LC}\left(\mathbb{R}^{n}\right)$ and $\operatorname{Cvx}\left(\mathbb{R}^{n}\right)$. More precisely we have

Theorem 22. Any bijective mapping $S: \operatorname{LC}\left(\mathbb{R}^{n}\right) \rightarrow \operatorname{Cvx}\left(\mathbb{R}^{n}\right)$ which preserves the (partial) order must be, up to a linear change, the support map $S$ defined above. more precisely, there exist constants $C_{0}, C_{1} \in \mathbb{R}$, vectors $v_{0}, v_{1} \in \mathbb{R}^{n}$ and a transformation $B \in \mathrm{GL}_{n}$ such that

$$
S(f)=C_{1}(\mathcal{L}(-\log f))\left(B x+v_{0}\right)+\left\langle x, v_{1}\right\rangle+C_{0} .
$$

Note that, since the mapping $I: \operatorname{LC}\left(\mathbb{R}^{n}\right) \rightarrow \operatorname{Cvx}\left(\mathbb{R}^{n}\right)$ given by $f \mapsto-\log f$ is an order reversing bijection, Theorem 22 is simply a restatement of the theorem mentioned in the introduction about order reversing bijective maps of convex functions (that they are essentially $\mathcal{L}$ ), which was demonstrated in [4]. Note also that for $S$ to map $\operatorname{LC}_{\mathrm{g}}\left(\mathbb{R}^{n}\right)$ onto $\mathrm{Cvx}_{0}\left(\mathbb{R}^{n}\right)$ (which is a natural requirement), one should require $v_{0}=v_{1}=0$ and $C_{0}=0$ as well.

More interestingly, our theorems imply that $M$ does not admit an order reversing extension to $\operatorname{LC}\left(\mathbb{R}^{n}\right)$ (under the inclusion $\mathcal{K}^{n} \subset \mathrm{LC}\left(\mathbb{R}^{n}\right)$ given by $K \mapsto 1_{K}$ ), and has a unique extension to a transform from $\mathrm{LC}_{\mathrm{g}}\left(\mathbb{R}^{n}\right)$ to $\mathrm{Cvx}_{0}\left(\mathbb{R}^{n}\right)$ which reverses the order of functions.

Theorem 23. There does not exist a bijective extension $\tilde{M}: \mathrm{LC}\left(\mathbb{R}^{n}\right) \rightarrow \operatorname{Cvx}\left(\mathbb{R}^{n}\right)$ of the Minkowski map (that is, such that $\left.\tilde{M}\right|_{\left\{1_{K}: K \in \mathcal{K}^{n}\right\}}=M$ ) which reverses the (partial) order. There exists an essentially unique order reversing extension of $M$ to $\tilde{M}: \mathrm{LC}_{\mathrm{g}}\left(\mathbb{R}^{n}\right) \rightarrow$ $\mathrm{Cvx}_{0}\left(\mathbb{R}^{n}\right)$ and it is, up to a linear change, the map $\tilde{M}(f)=\mathcal{J}(-\log f)$. 
Indeed, by Theorem 2 there are essentially only two transforms between $\mathrm{LC}_{\mathrm{g}}\left(\mathbb{R}^{n}\right)$ and $\operatorname{Cvx}_{0}\left(\mathbb{R}^{n}\right)$ which preserve order: $f \mapsto \mathcal{L}(-\log f)$ and $f \mapsto \mathcal{A}(-\log f)$. Thus, an order reversing transformation between $\mathrm{LC}_{\mathrm{g}}\left(\mathbb{R}^{n}\right)$ and $\operatorname{Cvx}_{0}\left(\mathbb{R}^{n}\right)$ is either essentially of the form $f \mapsto \mathcal{L} \mathcal{L}(-\log f)=-\log f$, which does not extend the operation $M$ from the class of convex sets, or essentially of the form $f \mapsto \mathcal{L} \mathcal{A}(-\log f)=\mathcal{J}(-\log f)$, which when restricted to indicator functions of bodies in $\mathcal{K}^{n}$, is equal to $M$ (and also equals $\mathcal{A} S(f)$ ). Similarly by Theorem 2 the only order reversing transform between $\operatorname{Cvx}\left(\mathbb{R}^{n}\right)$ and $\operatorname{LC}\left(\mathbb{R}^{n}\right)$ is $f \mapsto-\log f$, which does not extend $M$.

\subsection{Some calculus of these maps}

We end this section with a few simple examples of the behavior of the extensions of the support and Minkowski maps for geometric convex functions.

The support map is additive with respect to Asplund product (which we consider to be Minkowski addition for functions, see [2], with Prekopa-Leindler being exactly BrunnMinkowski for this addition). That is, defining

$$
(f \star g)(x)=\sup _{\{y+z=x\}} f(y) g(z)
$$

(again regularizing if the result is not upper-semicontinuous) we have

$$
S(f \star g)=S f+S g .
$$

The support function of a "convex set" is the usual dual norm (that is, $S$ extends the usual support operation):

$$
S\left(1_{K}\right)=\mathcal{L}\left(-\log 1_{K}\right)=\|\cdot\|_{K}^{*} .
$$

Similarly, $M$ extends the Minkowski functional,

$$
M\left(1_{K}\right)=\|\cdot\|_{K} \cdot
$$

When applied to $\mathcal{L} 1_{K}$, the support function returns $1_{K^{\circ}}^{\infty}$ :

$$
S\left(e^{-\|\cdot\|}\right)=1_{K^{\circ}}^{\infty}
$$

The Minkowski functional of a function $f$ is the support of the "geometrically dual" function $f^{\odot}$ (similarly to the fact that the Minkowski functional of a dual body is the support function of the original body),

$$
M(f)=\mathcal{A} S(f)=\mathcal{A L}(-\log f)=\mathcal{L} \mathcal{A}(-\log f)=S f^{\odot} .
$$

For the operation of g-inf-convolution we have

$$
M\left(e^{-(\phi \boxminus \psi)}\right)=\mathcal{J}(\phi \boxminus \psi)=\mathcal{J} \phi \square \mathcal{J} \psi=M\left(e^{-\phi}\right) \square M\left(e^{-\psi}\right) .
$$

This can be compared with

$$
S\left(e^{-(\phi \square \psi)}\right)=\mathcal{L}(\phi \square \psi)=\mathcal{L} \phi+\mathcal{L} \psi=S\left(e^{-\phi}\right)+S\left(e^{-\psi}\right) .
$$


Added in proof. 1. We learned very recently that the definition of the map $\mathcal{A}$ was given in the book by Rockafeller [15 p. 136]. In that book it is also proved that the transform commutes with the Legendre transform, a geometric interpretation of the transform through the epigraph is given (which is one of the two interpretations we consider), and a few simple examples are calculated. No uniqueness characterization is considered there.

2. We learned from Prof. S. S. Kutateladze that in the early ' 90 s there was an attempt to develop a theory of abstract convexity (see for example [16] and references therein), and in particular some abstract form of duality. The definition of abstract duality which was used was the exchange of infimum and supremum. However, to our knowledge no characterization of duality in any concrete case, classical or not, was achieved or even considered.

Acknowledgments. We would like to thank Dan Florentin for proofreading the text and providing useful remarks.

This research was supported in part by the Israel Science Foundation (first author: grant No. 865/07, second author: grant No. 387/09). Both authors were also partially supported by BSF grant No. 2006079.

\section{References}

[1] Artstein-Avidan, S., Florentin, D., Milman, V.: Preprint

[2] Artstein-Avidan, S., Klartag, B., Milman, V.: The Santaló point of a function, and a functional form of the Santaló inequality. Mathematika 51, 33-48 (2004) Zbl 1121.52021 MR 2220210

[3] Artstein-Avidan, S., Milman, V.: A characterization of the concept of duality. Electron. Res. Anounc. Math. Sci. 14, 42-59 (2007) Zbl 1140.52300 MR 2342714

[4] Artstein-Avidan, S., Milman, V.: The concept of duality in asymptotic geometric analysis, and the characterization of the Legendre transform. Ann. of Math. 169, 661-674 (2009) Zbl 1173.26008 MR 2480615

[5] Artstein-Avidan, S., Milman, V.: The concept of duality for measure projections of convex bodies. J. Funct. Anal. 254, 2648-2666 (2008) Zbl 1145.26003 MR 2406688

[6] Ball, K.: Logarithmically concave functions and sections of convex sets in $\mathbb{R}^{n}$. Studia Math. 88, 69-84 (1988) Zbl 0642.52011 MR 0932007

[7] Borell, C.: Convex set functions in $d$-space. Period. Math. Hungar. 6, 111-136 (1975) Zbl 0274.28009 MR 0404559

[8] Böröczky, K., Schneider, R.: A characterization of the duality mapping for convex bodies, Geom. Funct. Anal. 18, 657-667 (2008) Zbl 1168.52002 MR 2438994

[9] Brunn, H., Referat über eine Arbeit: Exacte Grundlagen für eine Theorie der Ovale. Sitzungsber. Math.-Phys. Cl. Akad. Wiss. München 24, 93-111 (1894) JFM 25.0873.03

[10] Gruber, P.: The endomorphisms of the lattice of convex bodies. Abh. Math. Sem. Univ. Hamburg 61, 121-130 (1991) Zbl 0754.52006 MR 1138277

[11] Gruber, P.: The endomorphisms of the lattice of norms in finite dimensions. Abh. Math. Sem. Univ. Hamburg 62, 179-189 (1992) Zbl 0779.52006 MR 1182848

[12] Klartag, B.: Marginals of geometric inequalities, In: Geometric Aspects of Functional Analysis, Lecture Notes in Math. 1910, Springer, 133-166 (2007) Zbl 1132.52015 MR 2349606

[13] Klartag, B., Milman, V.: Geometry of log-concave functions and measures. Geom. Dedicata 112, 169-182 (2005) Zbl 1099.28002 MR 2163897

[14] Milman, V.: Geometrization of probability. In: Geometry and Dynamics of Groups and Spaces, Progr. Math. 265, M. Kapranov et al. (eds.), Birkhäuser, 647-667 (2008) Zbl 1154.60022 MR 2402417 
[15] Rockafeller, R. T.: Convex Analysis. Princeton Univ. Press (1970) Zbl 0193.18401 MR 0274683

[16] Rubinov, A. M.: Abstract Convexity and Global Optimization. Nonconvex Optim. Appl. 44, Kluwer (2000) Zbl 0985.90074 MR 1834382

[17] Schneider, R.: The endomorphisms of the lattice of closed convex cones. Beiträge Algebra Geom. 49, 541-547 (2008) Zbl 1157.52004 MR 2468074 